\title{
Vis-NIR Disk-integrated Photometry of Asteroid 25143 Itokawa Around Opposition by AMICA/Hayabusa
}

Eri Tatsumi, Dept. Earth and Planetary Science, The Univ. of Tokyo, 7-3-1 Hongo, Bunkyo, Tokyo 113-0033 Japan

Deborah Domingue, Planetary Science Institute, 1700 E. Fort Lowell, Suite 106, Tucson AZ 85719-2395 USA

Naru Hirata, School of Computer Science and Engineering, The Univ. of Aizu, 90

Kami-Iwase, Tsuruga, Ikki-machi, Aizu-Wakamatsu, Fukushima 965-8580 Japan

Kohei Kitazato, School of Computer Science and Engineering, The Univ. of Aizu, 90

Kami-Iwase, Tsuruga, Ikki-machi, Aizu-Wakamatsu, Fukushima 965-8580 Japan

Faith Vilas, Planetary Science Institute, 1700 E. Fort Lowell, Suite 106, Tucson AZ

85719-2395 USA

Susan Lederer, NASA Johnson Space Center, Houston TX 77058

Paul R. Weissman, Planetary Science Institute, 1700 E. Fort Lowell, Suite 106, Tucson AZ 85719-2395 USA

Stephen C. Lowry, Centre for Astrophysics and Planetary Science, School of Physical Sciences, The University of Kent, Canterbury, CT2 7NH, UK

Seiji Sugita, Dept. Earth and Planetary Science, The Univ. of Tokyo, 7-3-1 Hongo, Bunkyo, Tokyo 113-0033 Japan; Research Center for the Early Universe, The Univ. of Tokyo, 7-3-1 Hongo, Bunkyo, Tokyo 113-0033 Japan 


\begin{abstract}
We present photometry of the S-type near-Earth asteroid 25143 Itokawa based on both ground-based observations in the UBVRI bands and measurements from the AMICA/Hayabusa spacecraft observations with ul-, b-, v-, w-, $\mathrm{x}_{-}^{-}$, and p-filters. Hayabusa observed Itokawa around opposition during the rendezvous, thus providing a unique set of observations of this asteroid. We fit the phase curve measurements with both the Classic Hapke model (Hapke, 1981, 1984, 1986) and Modern Hapke model (Hapke, 2002, 2008, 2012a) and thereby extract the physical properties of Itokawa's surface regolith. The single-scattering albedo $(0.57 \pm 0.05)$ is larger than that derived for Eros $(0.43 \pm 0.02)$, another S-type near-Earth asteroid visited by a spacecraft. Both models indicate a regolith that is forward-scattering in nature. From the hockey stick relationship derived for the single-particle phase function (Hapke, 2012b), both modeling results suggests a regolith comprised of rough surfaced particles with a low density of internal scatterers. Application of the Modern Hapke model derives porosity parameter values from 1 to 1.1, for BVR bands, which corresponds to porosity values between $77-79 \%$. This suggests the surface of Itokawa is very fluffy and the large boulders may be bonded with smaller size particles, typical of the particle sizes observed in the Muses Sea. Both models also provide similar geometric albedo values $(0.27 \pm 0.02)$ at the $\mathrm{V}$-band wavelength, which are equivalent to Eros' geometric albedo.
\end{abstract}




\subsection{Introduction}

The S-type asteroid 25143 Itokawa was observed by the Japanese spacecraft Hayabusa in 2005 (Fujiwara et al., 2006). The Hayabusa spacecraft, launched in 2003, rendezvoused with Itokawa in 2005 and successfully returned material from its surface to Earth in 2010 (e.g., Nagao et al., 2011). Remote sensing observations were made of the asteroid during the approach and rendezvous phases of the mission in order to provide information for the sample site selection and to place the sampling results in context with the broader surface. Among the remote sensing observations were images acquired by the Asteroid Multi-band Imaging CAmera (AMICA) and spectra by the Near InfraRed Spectrometer (NIRS).

Asteroid surfaces are understood to be covered by various-sized regolith grains based on ground-based photometry, thermal infrared observations, and spacecraft imaging data. The AMICA acquired images of Itokawa's surface display a wide variety of regolith types, especially in terms of regolith grain sizes and size distributions across its surface (Figure 1). The imaging data, taken at several wavelengths, provides broadband spectral information, photometric observations, and geologic imagery across Itokawa's surface. This study focuses on the photometric information, and examines the predictive capabilities of disk-integrated photometry for describing global regolith properties.

Over the past several decades, many asteroid flyby, orbital, and rendezvous missions have successfully acquired disk-resolved observations (e.g., Clark et al., 2002; Li et al., 2006; Magrin et al., 2012; Schröder et al., 2013). The Galileo mission flew by asteroids 951 Gaspra and 243 Ida, acquiring the first disk-resolved images of asteroids and their surfaces (Belton et al., 1992, 1994). The Galileo Solid State Imager (SSI) camera imaged Gaspra and Ida at phase angles of $33^{\circ}-51^{\circ}$ and $19^{\circ}-110^{\circ}$, respectively (Helfenstein et al., 1994, 1996). However, these observations did not include images near opposition (phase angle $<5^{\circ}$ ). Photometric observations of the asteroid 433 Eros were obtained by the NEAR-Shoemaker spacecraft at phase angles ranging from $1.2^{\circ}-111.0^{\circ}$ (Clark et al., 2002; Domingue et al., 2002; Li et al., 2004), well within the opposition surge. More recently, the Rosetta spacecraft flew by asteroids 2867 Steins, in 2008, and 21 Lutetia, in 2010. The imaging observations of Steins and Lutetia were acquired at phase angles of $0.36^{\circ}-141^{\circ}$ and $0^{\circ}-157^{\circ}$, respectively (Spjuth et al., 2012; Magrin et al., 2011; Masoumzadea et al., 2015), both containing observations well within the opposition region.

The opposition effect is the strong peak in the intensity of the reflected light from an object or surface around zero degrees phase angle. It was first observed in Saturn's rings by Seeliger (1895) and is also observed on the Moon (e.g. Hapke et al.,1993). The first observation of the opposition effect on an as asteroid was made in 1955 on the asteroid 20 Massalia (Gehrels, 1956). There are two contributing mechanisms for the opposition effect; shadow hiding and coherent backscattering (Hapke, 2012a). The shadow hiding opposition effect (SHOE) is a mechanism that occurs within media in which the particles are much larger than the observing wavelength. With the SHOE mechanism particles and surface structures cast larger and larger shadows on each other as the phase angle increases, thus less of the surface is illuminated to reflect light. The coherent backscattering opposition effect (CBOE) is prominent within media in which the particles are of the order of the wavelength in size and have a high single-scattering albedo. With the CBOE mechanism photons travel the same path, but in opposite directions, such that the photons add coherently upon reflection. 
Photometry models, such as Hapke's model (e.g. Hapke 2012a), have been applied to photometric measurements in an attempt to extract the physical characteristics of planetary surfaces, including asteroids. Laboratory and simulation tests of the correlation between Hapke model parameters with sample characteristics have shown evidence that the Hapke model parameters can be qualitatively related to physical properties, though quantitative correlations remain elusive (Shepard and Helfenstein 2007, Helfenstein and Shepard 2011, Souchon et al. 2011). For example, Helfenstein (1988) and Shepard and Campbell (1998) examined the roughness parameter using a computer generated rough surface, in which they found a correlation between the artificial rough surface and the Hapke's roughness parameter. A 'blind test' laboratory study, however showed only qualitative correlations between sample properties and model parameter values when using the most recent version of the Hapke model with an explicit porosity term (Shepard and Helfenstein 2007, Helfenstein and Shepard 2011).

Another example of the correlation between model parameters and regolith properties has been shown with the use of the empirical two parameters of the Henyey-Greenstein function to describe the single-particle phase function. Experimental studies measured the single scattering function for particles of different structures, which were fitted with the Henyey-Greenstein function to derive a relationship between single-particle phase function parameters and grain characteristics (e.g., McGuire and Hapke, 1995). Incorporating these measurements with applications to multiple solar system bodies, Hapke (2012b) found an empirical relationship (called the "hockey stick" relationship), which correlates Henyey-Greenstein function parameter values with general grain characteristics.

Moreover, of importance are measurements acquired within the opposition region, as these have been theoretically correlated to such surface properties as grain size and regolith porosity (Naranen et al., 2004; Shepard and Helfstein, 2007; Souchon et al., 2011). The SHOE mechanism (Hapke 1986) was incorporated into the Hapke model in its early iterations, but the CBOE mechanism was incorporated over a decade later (Hapke 2002). As will be discussed in Section 3, the earlier version of the Hapke model will be referred to as the "Classic Hapke Model" and the more recent version that includes the CBOE and additional porosity terms will be referred to as the "Modern Hapke Model". However, A quantitative correlation with regolith properties has not been firmly established on the real surfaces of asteroids. Application of the same model to different surfaces, where the properties could be examined with other methods, provides a mechanism for comparisons.

Clark et al. (2002) fit the NEAR's Near-Infrared Spectrometer's (NIS) photometric observations using the Classic Hapke Model. They found a decrease in the angular width of the opposition surge with increasing wavelength, which they interpreted to indicate a possible contribution from the CBOE. Spjuth et al. (2012) applied the Modern Hapke Model to the phase curve of E-type asteroid Steins with phase angle coverage between $0.36^{\circ}$ $141^{\circ}$. Their results are suggestive of a surface regolith with a large porosity $(\sim 84 \%)$ and moderate roughness $\left(\sim 28^{\circ}\right)$. However, the image data was of insufficient spatial resolution to resolve the surface regolith and verify the photometrically derived predictions of its properties.

This disk-integrated study is being used as a test case for examining the ability to sufficiently predict regolith properties in the early approach phases of sampling or landing missions, such as Hayabusa2 and OSIRIS-REx, at a time where sampling and landing strategies are first being initiated. The mechanical and optical system of the ONC (Optical 
Navigation Camera) onboard Hayabusa2 is nearly identical to AMICA (Suzuki et al, 2018), thus this provides a strong test for the early planning stage for Hayabusa2's approach to Ryugu. During close encounter missions, imaging acquired during the early phase are typically of low spatial resolution and often incorporate ground-based observations. Higher spatial resolution observations are acquired in the later mission phases, often as part of the approach to the landing/sampling site. This study evaluates the ability to estimate surface regolith properties by using photometric modeling techniques during the early phase, when only low resolution or point source images are available, to provide viable inputs to the landing/sampling strategies to an unknown surface. The goal of this study is to extract the physical characteristics of Itokawa's surface from the disk-integrated photometry observations and examine how well they correlate to the imaging observations acquired later in the mission and to the returned samples. To achieve this goal, we combine both ground-based and spacecraft observations to create the disk-integrated phase curves with the largest phase angle coverage possible. The Hayabusa AMICA images provide unique measurements within the opposition surge at multiple wavelengths while the ground-based measurements provide coverage beyond phase angle $100^{\circ}$. A by-product of the data combination is a verification of the AMICA calibration. We examine the applicability of both the Classic and Modern Hapke models, comparing predicted morphologies with those based on the high-resolution images taken by AMICA and other information derived from sample analyses and remote-sensing observations.
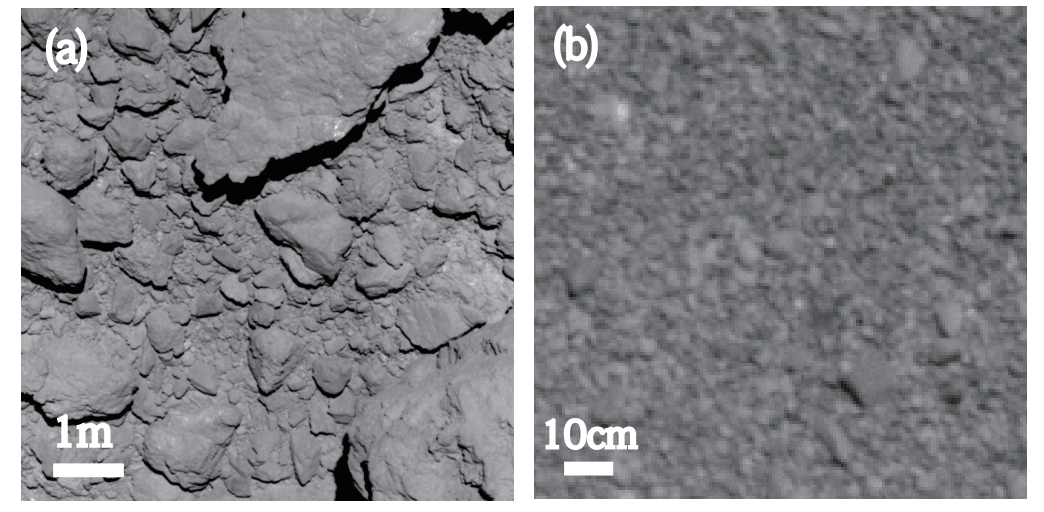

Figure 1. Images of Itokawa's surfaces with different grain sizes. A surface covered with coarse gravels (a) st_2539437177_v and a surface covered with fine pebbles at Muses Sea (b) st_2539437177_v. These image file names correspond to the PDS file names.

\section{Datasets and data reduction}

\subsection{AMICA (Asteroid Multi-band Imaging CAmera)}

AMICA was the multiband CCD imager onboard the Hayabusa spacecraft (Ishiguro et al., 2010). It contained 7 color filters (ul: $381 \mathrm{~nm}$, b: $429 \mathrm{~nm}$, v: $553 \mathrm{~nm}, \mathrm{w}: 700 \mathrm{~nm}$, x: $861 \mathrm{~nm}$, p: $960 \mathrm{~nm}$, zs: $1008 \mathrm{~nm}$ ), corresponding to the Eight Color Asteroid Survey (ECAS) by Zellner et al. (1985), and a wide-band filter. AMICA acquired more than 1400 images of Itokawa during its approach and rendezvous with Itokawa, covering a phase angle range between $0.7^{\circ}-37^{\circ}$ (Figure 2). The detailed system properties of AMICA are described in Ishiguro et al. (2010). We used 347 AMICA images obtained with the ul-, b-, v-, w-, x-, and p-filters to construct the disk-integrated phase curves of Itokawa in visible wavelengths 
(Table. 1). All the images used in this study are archived in the Planetary Data System (PDS) https://sbn.psi.edu/pds/resource/hayamica.html).

(HAY-A-AMICA-3-HAYAMICA-V1.0,

We used only uncompressed images that included the whole disk of Itokawa. Because the archived data are raw images, we applied both a smear and flat-field correction using the same procedure as described by Ishiguro et al. (2010). The halo around the disk spread over a considerably large region, especially for $\mathrm{x}$ - and $\mathrm{p}$-filters, which is due to scattered light effects within the optical system, as reported by Ishiguro (2014). Because of the scattered light effect, we carefully chose images that included both the disk and its halo.

The total light flux $Q_{n}$ from the asteroid is calculated by integrating the intensity I of the region of interest (ROI) which includes the entire disk of Itokawa and the scattered light halo.

$$
\begin{aligned}
& Q_{n}=\frac{C_{0} C_{n}\left(S_{n} / S_{v}\right)}{t_{\text {exp }}} \sum_{\text {ROI }} I(H, V)\left[W m^{-2} \mu m^{-1} s r^{-1} p i x\right] \quad E q(2.1) \\
& (n=u l, b, v, w, x, p)
\end{aligned}
$$

where, $I(H, V)[D N]$ is the intensity of each pixel within the AMICA CCD coordinate system, $C_{0}$ is the calibration factor for v-band $(3.42 \pm 0.10) \times 10^{-3}\left[\mathrm{Wm}^{-2} \mu \mathrm{m}^{-1} \mathrm{sr}^{-1}\right] /\left[\mathrm{DNs} \mathrm{s}^{-1}\right], C_{n}$ is the scale factor for each band (Ishiguro et al., 2010), $S_{n}$ is the solar irradiance spectrum at $1 \mathrm{AU}$ (http://rredc.nrel.gov/solar/spectra/am0/ASTM2000.html). The radiance factor is the ratio of the total light flux from lambertian surface $Q_{\text {sun }}$ and the total light flux from the object $Q_{n}$.

$$
\left(\frac{I}{F}\right)_{n}=\frac{Q_{n}}{Q_{\text {sun } n}}=\frac{Q_{n}}{S_{n} /\left(\pi r^{2}\right) A} \quad E q(2.2)
$$

where, $r$ is the heliocentric distance, $A$ is the projected pixel area of Itokawa from the direction of AMICA at the time of observation calculated based on the SPICE kernels and the shape model (hay-a-spice-6-v1.0, HAY-A-AMICA-5-ITOKAWASHAPE-V1.0).

In order to combine the AMICA derived data with the ground-based measurements, we had to take into account the transmittance difference of each of the filters (Figure 3). We calculated conversion factors from the AMICA-filter system ( $u l, b, v, w, x, p)$ to the UBVRI system. We estimated the discrete spectrum for each system to multiply the transmittance function and the continuous spectrum obtained by Lowry et al. (2005) (Figure 4). We obtained the conversion factor by taking the ratio of these discrete spectra. Note that there is no corresponding ground-based data to p-filter, however the opposition measurements in this filter encouraged us to include it in our study. The conversion factors for the AMICA filters to the UBVRI system are given by:

$$
\begin{aligned}
& I_{U}=1.14 I_{u l} \\
& I_{B}=1.04 I_{b} \\
& I_{V}=1.00 I_{v} \\
& I_{R}=0.96 I_{w} \\
& I_{I}=1.07 I_{x}
\end{aligned}
$$

Table 1 AMICA images used in this study. 


\begin{tabular}{|l|l|l|}
\hline $\begin{array}{l}\text { Band name } \\
\text { (wavelength) }\end{array}$ & Phase angle (deg) & Number of images \\
\hline ul (380 nm) & $7.7-23.5$ & 8 \\
\hline b (429 nm) & $0.7-36.9$ & 56 \\
\hline v (553 nm) & $0.7-30.3$ & 135 \\
\hline w (700 nm) & $0.7-20.2$ & 71 \\
\hline x (861 nm) & $7.7-8.6$ & 5 \\
\hline p (960 nm) & $0.7-36.8$ & 72 \\
\hline
\end{tabular}

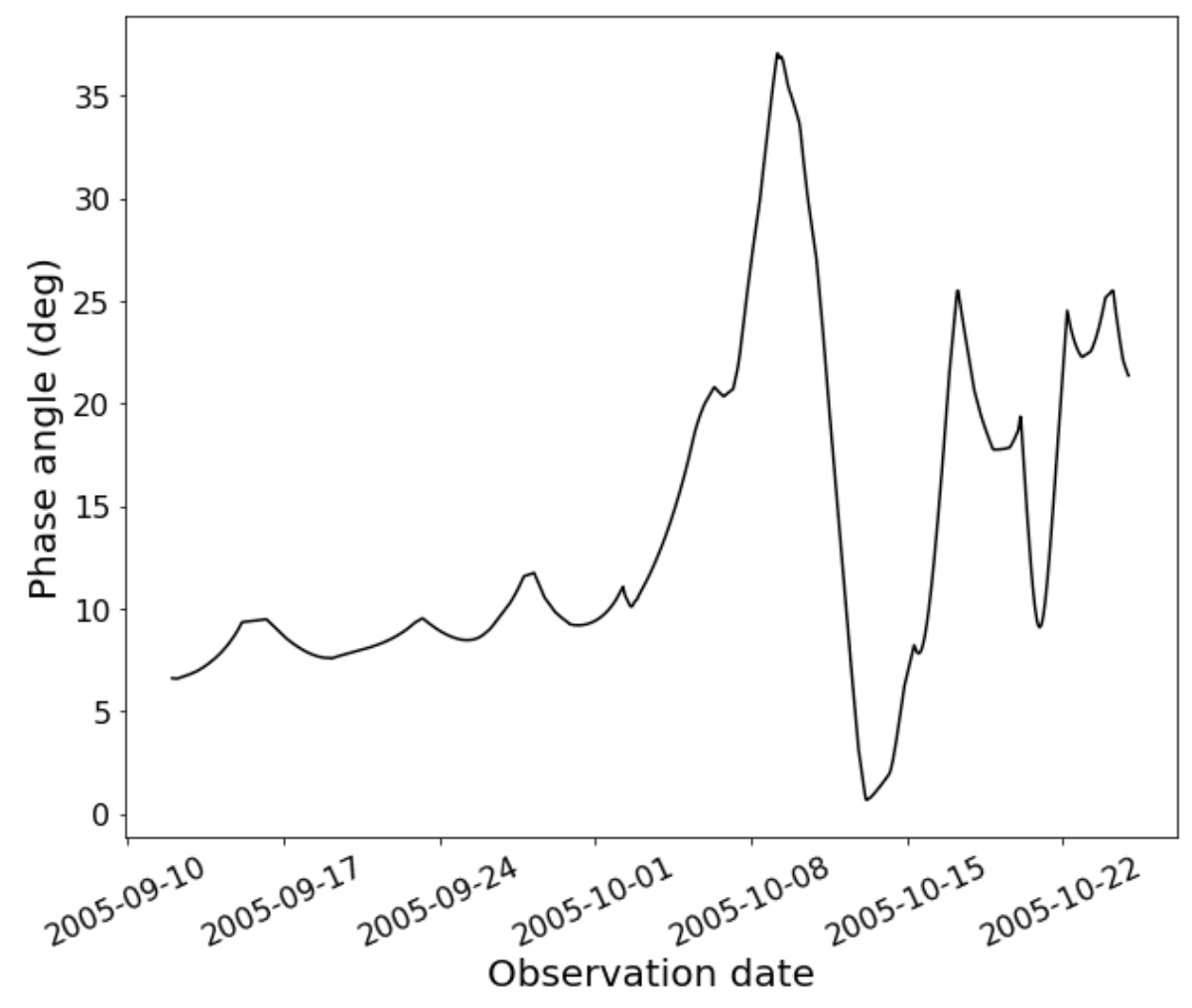




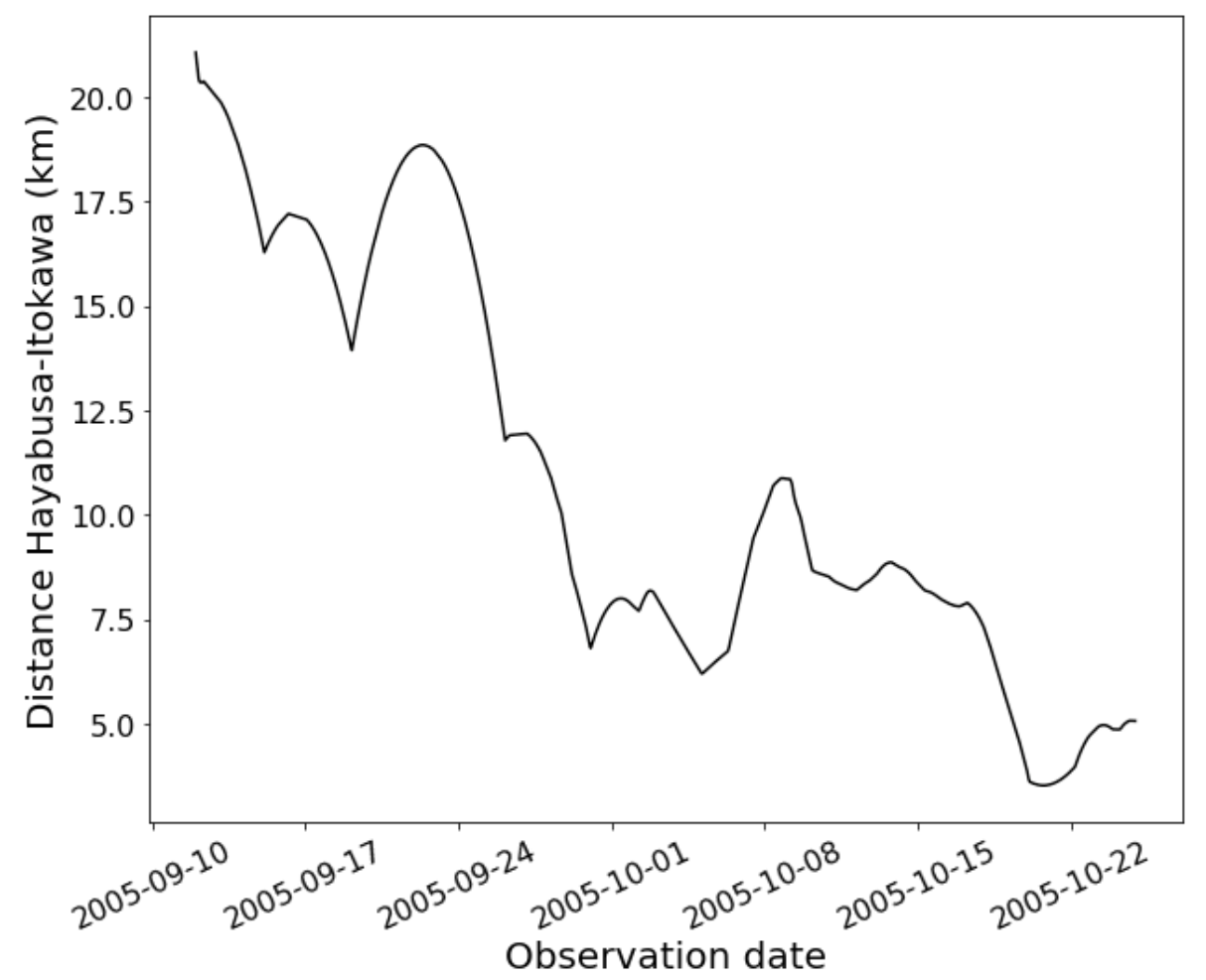

Figure 2. Phase angle (a) and distance (b) during the rendezvous of Hayabusa with the target asteroid Itokawa.

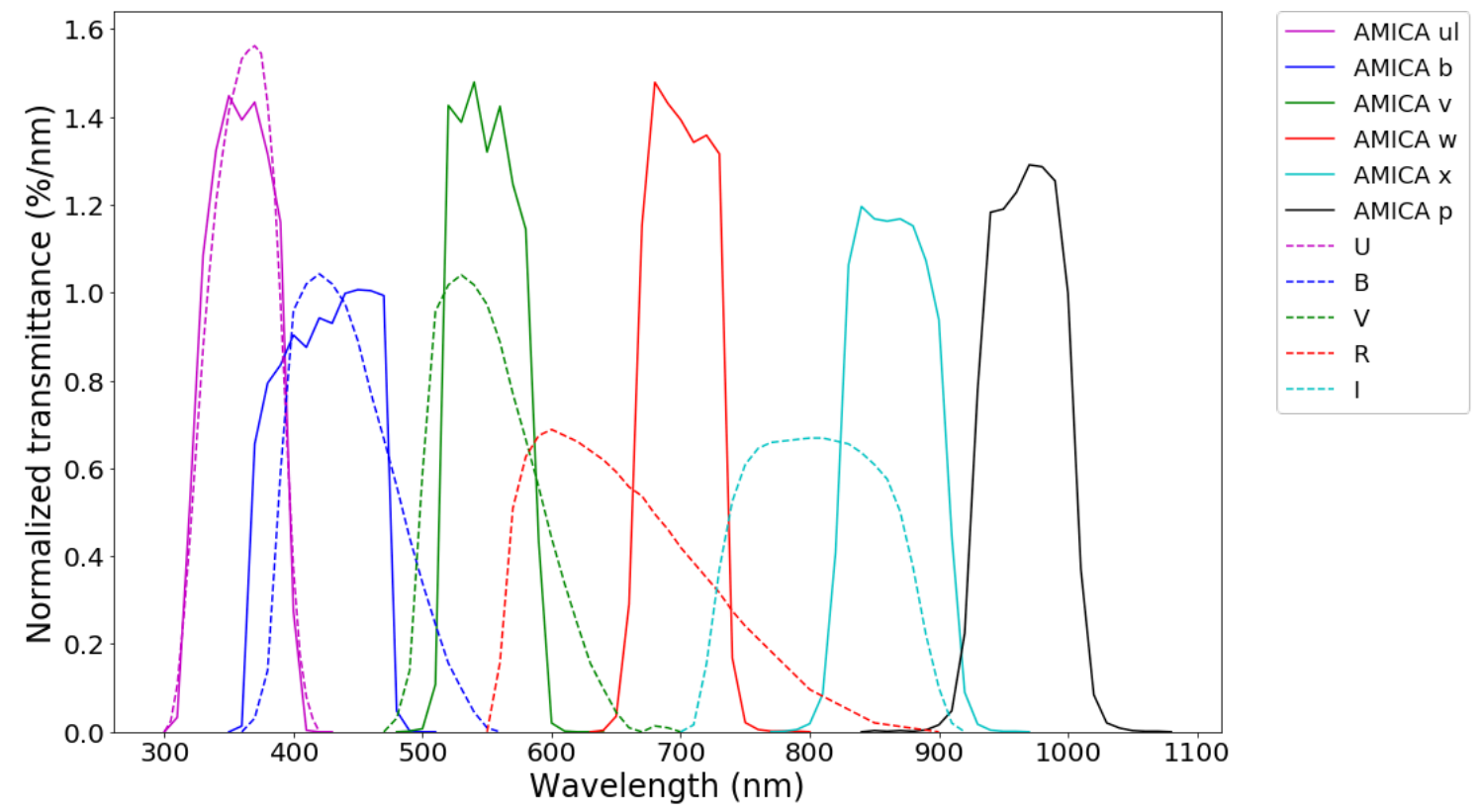

Figure 3. Comparison of normalized filter transmittance functions of the AMICA system and the UBVRI system. The transmittance functions are normalized so that the integral of each function over wavelength is unity. 


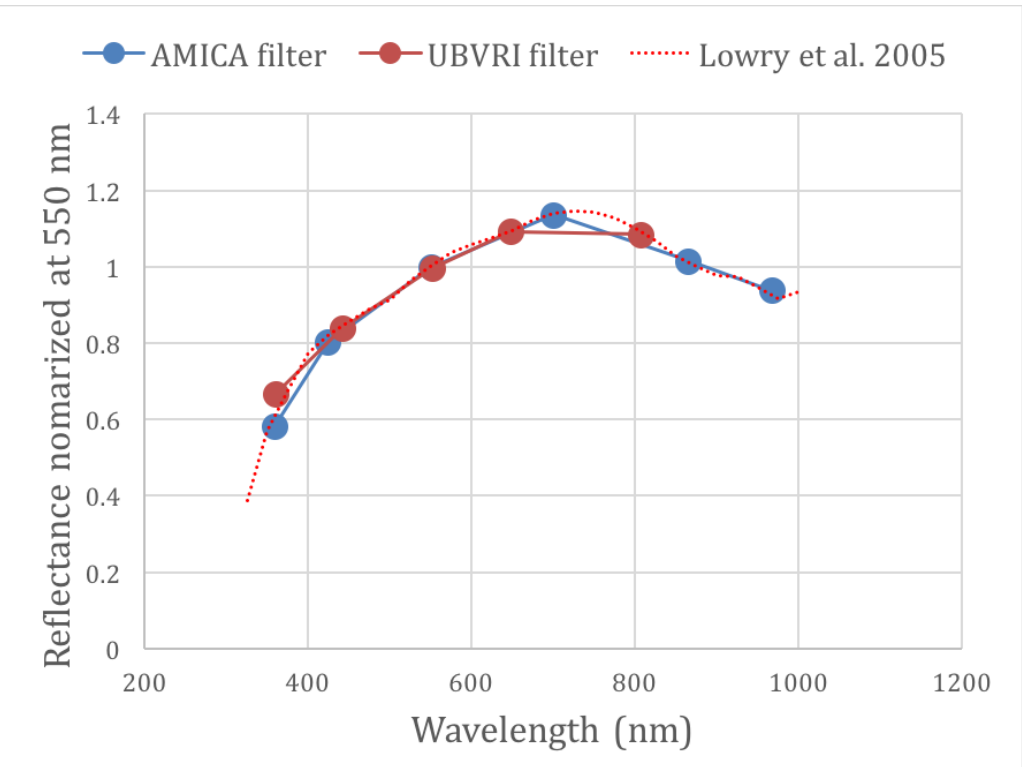

Figure 4 Comparison of Itokawa discrete spectra of the AMICA system and the UBVRI system calculated from continuous spectra by Lowry et al. (2005).

\subsection{AMICA phase curves}

As will be discussed in the next section, the ground-based phase curves used in this analysis incorporate average values from daily observations. In the case of the AMICA derived measurements, averages were taken at every $\sim 0.1^{\circ}$ between $6.5^{\circ}$ and $9.5^{\circ}$ where the data are clustered. Average values were calculated for both phase angle and reflectance values. Measurements taken at all other phase angles were incorporated into the phase curve without any modification. The resulting data set is referred to as the 'averaged' data set. The complete AMICA v-band data are compared with the 'averaged' data set in Figure 5a. This method of averaging was performed for all the AMICA data sets.

The AMICA data were combined with the ground-based observations presented by Lederer et al. (2008) at corresponding wavelengths, where available. The combined data at $540 \mathrm{~nm}$ (corresponding to the AMICA v-band) are displayed in Figure $\mathbf{5 b}$ as an example. The phase curve for all the data sets are heavily populated at the smaller $\left(<50^{\circ}\right)$ phase angles, thus weighting the data set more strongly in the backward scattering direction outside of the opposition region. The B- $(440 \mathrm{~nm}), \mathrm{V}-(540 \mathrm{~nm})$, and R- $(630 \mathrm{~nm})$ filter data sets each contain a single measurement in the forward scattering region at $\sim 130^{\circ}$ phase. The U-filter $(360 \mathrm{~nm})$, I-filter $(850 \mathrm{~nm})$, and p-filter $(960 \mathrm{~nm})$ data sets do not contain any measurements in the forward scattering region (phase $>90^{\circ}$ ). In addition, the opposition data $\left(<4^{\circ}\right.$ phase) is present only for the BVR filters and the AMICA p-band data. In order to provide a data set that equally weighted all regions of the phase curve 'anchor points' were defined in the BVR data sets. These anchor points are defined as points within the averaged data set that are representative of the phase curve and spaced evenly in phase angle, as the data set allows (Figure 5b). The importance and use of these anchor points is discussed in more detail in Section 3.2 (Modeling Methodology). 

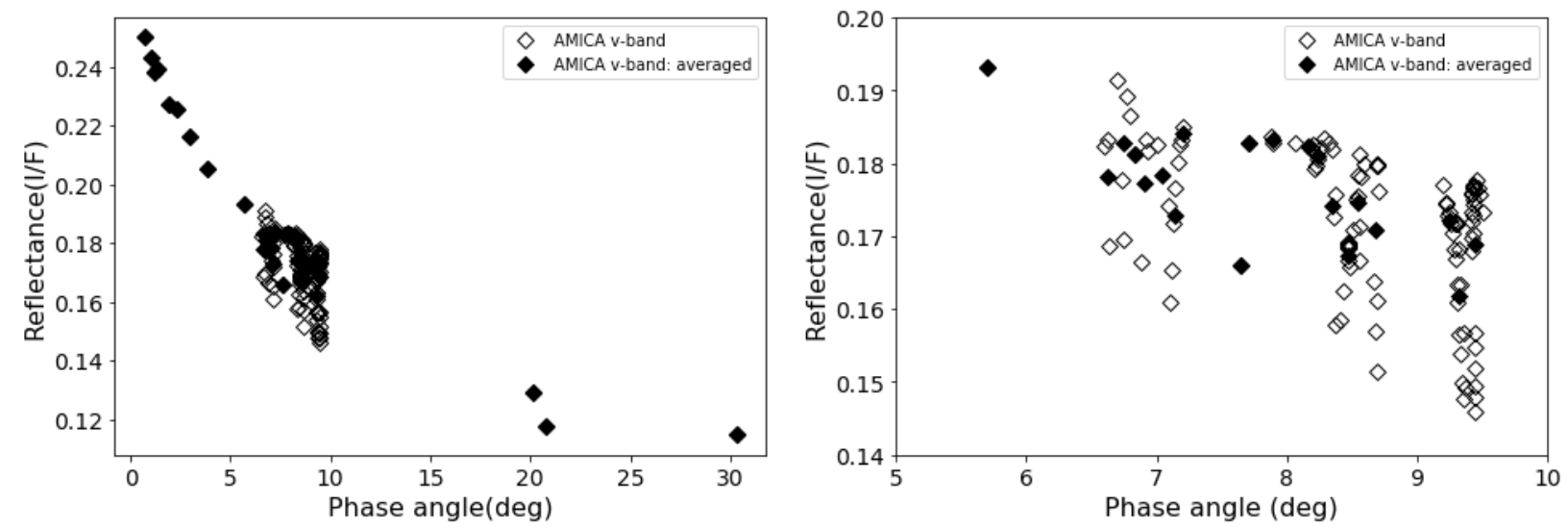

Figure 5a. These graphs display the AMICA v-band data, which corresponds in wavelength to the Johnson V-filter (540nm). The black, open diamonds denote the disk-integrated measurements extracted from each of the v-band images acquired by Hayabusa, where the entire disk of Itokawa is contained within the image frame. The black-filled diamonds represent the 'averaged' data set extracted from this data set.

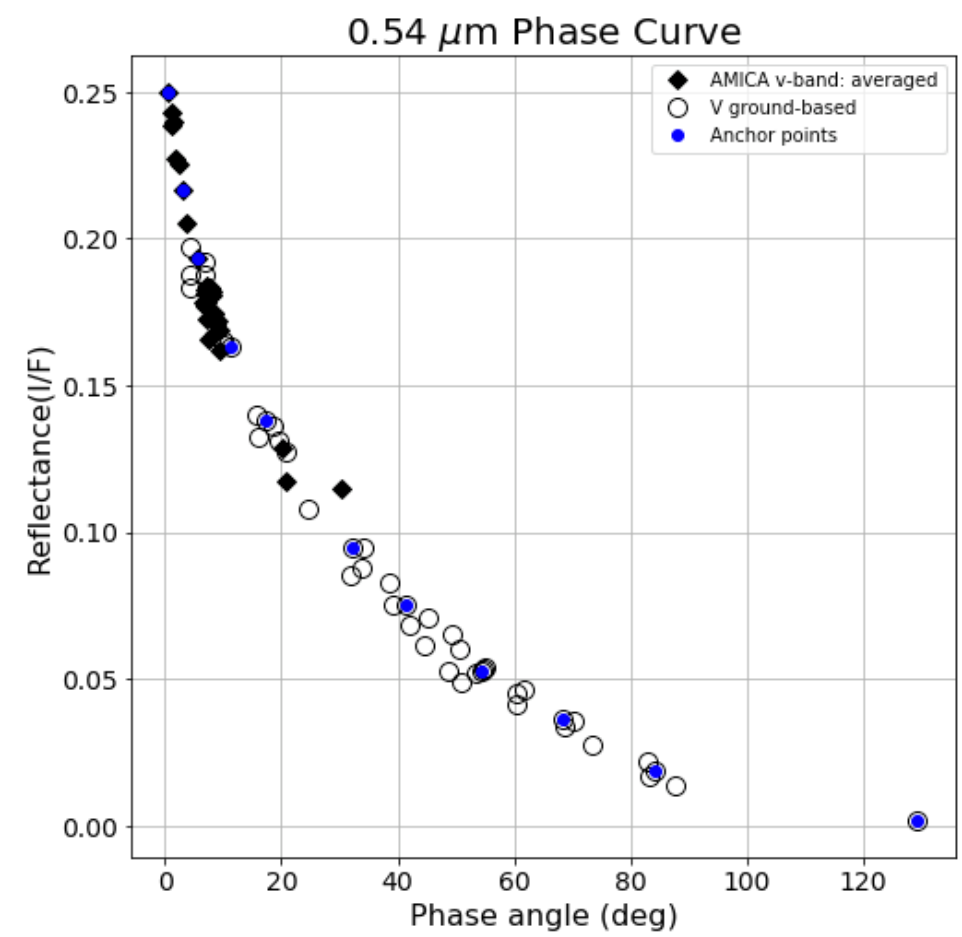

Figure 5b. This graph compares the ground-based $V$-filter observations (open circles) from Lederer et al. (2008) with the averaged AMICA v-band measurements (black diamonds). The anchor points for this combined data set are marked by blue circles.

\subsection{Ground-based observations}

Due to the lack of measurements at large phase angles from the AMICA images, we combined the spacecraft-based measurements with the ground-based observational data 
from Lederer et al. $(2005,2008)$. The ground-based data from Lederer et al. $(2005,2008)$ covers the phase angle range between $4^{\circ}-129^{\circ}$, and was acquired from several observatories with UBVRI broadband filters from January - September 2001. For details on the reduction of these observations the reader is referred to Lederer et al. $(2005,2008)$.

One modification made to the ground-based data for this study was the incorporation of the Itokawa shape model in calculating the projected area of Itokawa at the time of observation. The Lederer et al. $(2005,2008)$ observations were obtained and processed before Hayabusa's arrival at Itokawa. Thus, an ellipsoid model was used to calculate the projected area of Itokawa at the time of each observation. The detailed observations of Itokawa by AMICA instrument made it possible to create a very high-resolution shape model (HAY-A-AMICA-5-ITOKAWASHAPE-V1.0), available in the PDS archive. We recalculated the projected area using the shape model and the rotational period of $12.132371 \pm 6 \times 10^{-6} \mathrm{~h}$ (Lowry et al., 2014). Figure 6(a) shows an example of a simulated image from the shape model. Figure 6(b) shows the comparison of projected area calculated from the shape model used in this study and ellipsoid-based model used in Lederer et al. (2005). The projected area calculations accounted for orientations where, due to Itokawa's bi-lobed shape, portions of the asteroid shadowed other regions on the asteroid. Because this projected area made up to $\sim 20 \%$ difference in I/F value from the ellipsoid-based area calculations, we used the projected area calculated from the shape model to account for the effect of rotational phase. After correcting for rotational phase, daily averages were used for the photometric phase curve. This is to remove the variations due to local albedo differences and shadow caused by macroscopic roughness across the surface, as seen in the AMICA imagery. The combined AMICA and ground-based phase curves, along with the modeling solutions are shown in Figures $\mathbf{7}$ and 8.
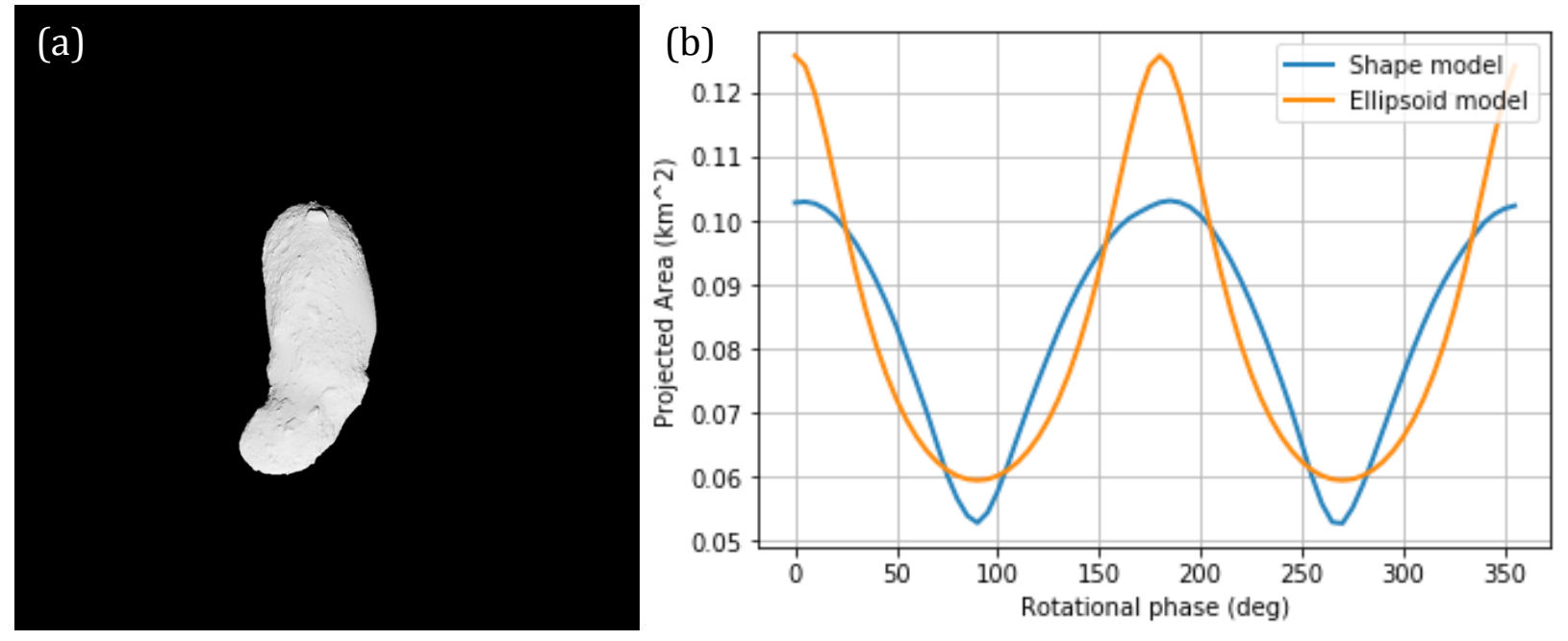

Figure 6. (a) Simulated Itokawa image from the high-resolution shape model (quad512q.tab, HAY-A-AMICA-5-ITOKAWASHAPE-V1.0) at phase angle 8.9 ${ }^{\circ}$. (b) Projected area comparison based on the shape model and the ellipsoid-based model. 


\subsection{Comparison with Hapke's Model}

UBVRI ground-based observations of Itokawa, prior to Hayabusa's encounter, were acquired by Lederer et al. $(2005,2008)$ and analyzed using the Classic Hapke Model. This model, given below, incorporates an opposition surge due to shadow hiding and a surface roughness term. The Modern Hapke Model, which incorporates an additional opposition surge term due to coherent backscatter (Hapke 2002, 2012a) and porosity (Hapke 2008, 2012) were not selected due to the paucity of opposition data below $5^{\circ}$ phase, and the later development of the model. For comparison purposes, we first fit the combined AMICA and ground-based disk-integrated phase curves using the same model as Lederer et al. (2005, 2008) and then again with the Modern Hapke Model (2012a). The detailed description of the models is in Appendix A.

\subsection{Modeling Methodology}

Both the Classic and Modern Hapke models are mathematically complex, where changes in the shape of the disk-integrated phase curve due to variations in one of the model parameter values can be negated or mimicked by changes in a different model parameter value. For example, the shadow-hiding amplitude parameter, $B_{s 0}$, if allowed to be greater than unity, can modify the model-derived reflectance beyond $20^{\circ}$ phase; well outside the opposition region. This will influence the corresponding values of both the SSA and the surface roughness parameter. Unevenly distributed measurements or observations along the phase curve (such as a larger percentage of data points at small to mid-range phase angles compared to large phase angles) can weigh the influence of one parameter over another in fitting the shape of the phase curve. In order to minimize the mathematical coupling between the parameters a step-wise strategy in applying the models was derived.

The wavelengths or filters with the most comprehensive combined data sets are the BVR filter data sets. They include opposition measurements from AMICA down to $0.69^{\circ}$ and ground-based observations out to $129^{\circ}$. Anchor points were defined for these data sets, where measurements were selected a single points along the phase curve to define the phase curve uniformly in phase angle, within the available measurements. The first step in the modeling strategy was to fit the models to just the anchor points. An example of the anchor point selection is shown in Figure 5b.

Parameter values were found utilizing a least-squares grid search, where each parameter was varied independently and concurrently. The solution methodology follows those used by Domingue et al. $(1991,1995,2002,2010)$ and Lederer et al. $(2005,2008)$, where the value of $\chi$ is minimized. $\chi$ is defined by

$$
\chi=\frac{\sqrt{\left(R_{\text {model }}-R_{\text {observed }}\right)^{2}}}{N}, \quad E q(3.7)
$$

where $N$ is the number of data points, $\mathrm{R}_{\text {model }}$ is the Hapke model predicted reflectance, and $R_{\text {observed }}$ is the measured reflectance from either the ground-based observations or the disk-integrated reflectance extracted from the AMICA images. The grid for each parameter 
was given an initial increment (grid size) to identify local minima in the parameter solution space. Each local minimum was then searched on a final grid size. Each local minimum was examined in terms of relative $w$ values between the different filters, and the difference in $\chi$ values between the parameter solutions of each local minima. The solutions were spectrally constrained to produce wavelength dependent $w$ values that matched the shape of the reflectance spectrum of Itokawa, with the caveat that the difference in $\chi$ value was less then $10^{-5}$ with the lowest $\chi$ value. The parameter sets with the top ten lowest $\chi$ values were stored for each run of the grid search. The error bars for the parameters are based on the range of values in the top ten sets of the final run plus the grid size. The range of values and grid sizes for each parameter, as applied to the anchor points, are given in Table 2.

The parameter values derived from fitting the anchor points were then used as initial inputs to fitting the entire data sets for the BVR filters. The values for the SSA and surface roughness were set to those found by fitting the anchor points and were not varied. In fitting the Classic Hapke model, the value for $B_{s 0}$ was varied by \pm 0.05 from the anchor point solution, the value of $h_{s}$ was varied by \pm 0.02 from the anchor point solution, and the single-particle phase function parameters $(b$ and $c$ ) were varied by \pm 0.1 from the anchor point solution. The same constraints were used in the application of the Modern Model, with $B_{c 0}$ and $h_{c}$ varied similarly as Bso and hs, respectively. Using the least-squares grid described above, with the final grid sizes listed in Table 2, the best-fit parameters to the combined AMICA and ground-based data set was determined.

The U-filter, I-filter, and p-filter data were modeled only with the Classic Hapke model, as these data do not include sufficient phase angle coverage to provide a reasonable constraint on all the parameters in the Modern Hapke model. The U- and I-filter data sets do not contain opposition measurements below phase angle $6^{\circ}$, and are constrained to measurements below phase angle $\sim 70^{\circ}$ and $\sim 84^{\circ}$, respectively (thus no constraints on the forward scattering direction). The p-filter data, derived solely from the AMICA images, contains measurements within the opposition region similar to the BVR coverage, however lacks any measurements beyond phase angle $\sim 37^{\circ}$. To fit these three data sets the following constraints were made on the range of values for the Classic Hapke model parameters:

- SSA values were constrained to mimic the spectral reflectance curve,

○ thus values for the U-filter were limited to be less than the B-filter value,

$\circ$ values for the I-filter were limited to be greater than the R-filter value,

$\circ$ values for the p-filter were limited to range between the V- and R-filter values

- the surface roughness parameter was set to the average value obtained for the BVR data sets (the values for these filters were equivalent within the error bars),

- the opposition parameter value ranges were based on the range of values obtained for the BVR data sets such that:

○ the $B_{s 0}$ range was set to the B-filter value or higher for the U-filter modeling

○ the $B_{s 0}$ range was set to the R-filter value or higher for the I-filter modeling

$\circ$ the $h_{s}$ range was set to the B-filter value or 0.01 higher for the U-filter modeling

○ the $h_{s}$ range was set to the R-filter value or 0.01 higher for the I-filter modeling 
○ the $B_{s 0}$ range was set to the R-filter value \pm 0.1 for the $\mathrm{p}$-filter modeling (not allowed to exceed unity)

$\circ$ the $h_{s}$ range was set to the R-filter value \pm 0.08 for the p-filter modeling (not allowed to be below 0.0 )

- scattering function parameters were set to \pm 0.1 of the minimum and maximum values for $\mathrm{b}$ and $\mathrm{c}$ over the BVR solutions.

Table 2. Least-Squares Grid Search Range and Search Increments

\begin{tabular}{|c|c|c|c|}
\hline $\begin{array}{c}\text { Model } \\
\text { Parameter }\end{array}$ & $\begin{array}{l}\text { Value } \\
\text { Range }\end{array}$ & $\begin{array}{c}\text { Grid Size } \\
\text { (initial - final) }\end{array}$ & Parameter Description \\
\hline \multicolumn{4}{|c|}{ Classic Hapke Model } \\
\hline$w$ & $0-1$ & $0.05-0.01$ & Single scattering albedo (SSA) \\
\hline$B_{S O}$ & $0-1$ & $0.1-0.01$ & $\begin{array}{l}\text { Opposition Effect Amplitude } \\
\text { (Shadow-Hiding (SHOE)) }\end{array}$ \\
\hline$h_{s}$ & $\begin{array}{c}0.001- \\
0.301\end{array}$ & $0.05-0.01$ & $\begin{array}{l}\text { Opposition Effect Width } \\
\text { (SHOE) }\end{array}$ \\
\hline$b$ & $0-1$ & $0.1-0.01$ & Single-particle phase function amplitude \\
\hline$c$ & $0-1$ & $0.1-0.01$ & $\begin{array}{l}\text { Single-particle phase function partition } \\
\text { parameter }\end{array}$ \\
\hline$\theta$ & $0^{\circ}-40^{\circ}$ & $5^{\circ}-1^{\circ}$ & Surface roughness (degrees) \\
\hline \multicolumn{4}{|c|}{ Modern Hapke Model } \\
\hline$w$ & $0-1$ & $0.2-0.01$ & Single scattering albedo (SSA) \\
\hline$B_{S O}$ & $0-1$ & $0.1-0.01$ & $\begin{array}{l}\text { Opposition Effect Amplitude } \\
\text { (Shadow-Hiding (SHOE)) }\end{array}$ \\
\hline$h_{s}$ & $\begin{array}{c}0.001- \\
0.301\end{array}$ & $0.05-0.01$ & $\begin{array}{l}\text { Opposition Effect Width } \\
\text { (SHOE) }\end{array}$ \\
\hline$B_{C O}$ & $0-1$ & $0.1-0.01$ & $\begin{array}{l}\text { Opposition Effect Amplitude } \\
\text { (Coherent-Backscatter (CBOE)) }\end{array}$ \\
\hline$h_{c}$ & $\begin{array}{c}0.001- \\
0.301\end{array}$ & $0.05-0.01$ & $\begin{array}{l}\text { Opposition Effect Width } \\
\text { (CBOE) }\end{array}$ \\
\hline$b$ & $0-1$ & $0.2-0.01$ & Single-particle phase function amplitude \\
\hline$c$ & $0-1$ & $0.2-0.01$ & $\begin{array}{l}\text { Single-particle phase function partition } \\
\text { parameter }\end{array}$ \\
\hline$\theta$ & $0^{\circ}-40^{\circ}$ & $5^{\circ}-1^{\circ}$ & Surface roughness (degrees) \\
\hline$k$ & $1-1.5$ & $0.2-0.02$ & Porosity \\
\hline
\end{tabular}

The disk-integrated modeling results were then tested and refined utilizing disk-resolved measurements. Using the shape model, the surface of Itokawa was re-created at a phase angle of $\sim 7.9^{\circ}$ phase angle, based on one of the images acquired by AMICA (Figure 10). The reflectance of the surface was calculated using both the Classic and Modern Hapke model values for each of the BVR solution sets. The reflectance values from both models were then compared. It was found that while both models produced highly similar disk-integrated phase curves, they produced drastically different reflectance values in the disk-resolved case. The surface based on the Modern Hapke model values was significantly brighter than the Classic Hapke model surface. 
The ratio between the reflectances from the Classic and Modern Hapke models was calculated (hereafter referred to as the "C/M ratio") for the variety of $(i, e, \alpha)$ over the above shape to constrain the value of $k$. In each case, the average and median value of the $\mathrm{C} / \mathrm{M}$ ratio over the entire surface was identical to within 0.001. Since the Modern Hapke model contains the larger number of free parameters, and is thus more difficult to constrain with just disk-integrated measurements, the Modern Hapke model was refit to the disk-integrated data with the porosity parameter $(k)$ set to $k$ times the median C/M ratio and all other parameters varying within \pm 0.1 of the initial solution. The $\chi$ values for the resulting solutions were within $1 \times 10^{-6}$ of the original $\chi$ values. These solutions were taken as the final Modern Hapke model parameter values, providing solutions commensurate with the Classic Hapke model values.

\subsection{Modeling Results}

The modeling results using both the Classic and Modern Hapke models are displayed and contrasted with the modeling results from Lederer et al. (2008), where available, in Figures 7 and 8. The Classic Hapke model parameter values from this study are compared with the Classic Hapke model results from Lederer et al. (2008) in Table 3. The parameter values for the Modern Hapke modeling results are displayed in Table 4. There are a few notable differences between the Lederer et al. (2008) parameter values and those derived in this study. The first is the generally lower (darker) SSA in this study's results, though both show a large $\left(>30^{\circ}\right)$ surface roughness. The single-particle phase function parameters are similar between the Classic and Modern Hapke modeling results from this study, but are less forward scattering than predicted by the Lederer et al. (2008) study.

In general, the largest difference between the two Classic model studies is in the opposition parameters. The data provided by AMICA in the opposition region, especially for the BVR wavelengths (Figure 7), provides the needed data to better constrain the model in this region of the phase curve. This study shows a stronger, narrower peak than the previous Lederer et al. (2008) results, which will be discussed in more detail in Section 5.1.

Table 3. Classic Hapke Model Parameter Values

\begin{tabular}{|c|c|c|c|c|c|c|c|c|c|c|c|}
\hline \multirow{2}{*}{$\begin{array}{c}\text { Classis } \\
\text { Hapke } \\
\text { Paramet } \\
\text { ers }\end{array}$} & \multicolumn{2}{|c|}{$\mathrm{U}(0.36 \mu \mathrm{m})$} & \multicolumn{2}{|c|}{$B(\mu m)$} & \multicolumn{2}{|c|}{$\mathrm{V}(0.54 \mu \mathrm{m})$} & \multicolumn{2}{|c|}{$\mathrm{R}(0.63 \mu \mathrm{m})$} & \multicolumn{2}{|c|}{$\mathrm{I}(0.85 \mu \mathrm{m})$} & \multirow{2}{*}{$\begin{array}{l}p(0.96 \\
\mu \mathrm{m}) \\
\text { This } \\
\text { Study }\end{array}$} \\
\hline & $\begin{array}{l}\text { Ledere } \\
\text { r et al. } \\
(2008) \\
\end{array}$ & $\begin{array}{l}\text { This } \\
\text { Study }\end{array}$ & $\begin{array}{l}\text { Ledere } \\
\text { r et al. } \\
(2008)\end{array}$ & $\begin{array}{l}\text { This } \\
\text { Study }\end{array}$ & $\begin{array}{c}\text { Ledere } \\
\mathrm{r} \text { et al. } \\
(2008)\end{array}$ & $\begin{array}{l}\text { This } \\
\text { Study }\end{array}$ & $\begin{array}{l}\text { Ledere } \\
\text { r et al. } \\
(2008)\end{array}$ & $\begin{array}{l}\text { This } \\
\text { Study }\end{array}$ & $\begin{array}{c}\text { Ledere } \\
\mathrm{r} \text { et al. } \\
(2008)\end{array}$ & $\begin{array}{l}\text { This } \\
\text { Study }\end{array}$ & \\
\hline \multirow{2}{*}{$w$} & $0.53 \pm$ & $0.43 \pm$ & $0.66 \pm$ & $0.51 \pm$ & $0.7 \pm$ & $0.57 \pm$ & $0.71 \pm$ & $0.60 \pm$ & $0.73 \pm$ & $0.64 \pm$ & $0.59 \pm$ \\
\hline & 0.07 & 0.02 & 0.04 & 0.04 & 0.04 & 0.05 & 0.04 & 0.05 & 0.04 & 0.03 & 0.03 \\
\hline \multirow{2}{*}{ Bso } & \multirow{2}{*}{$1^{*}$} & 0.99 & $0.04 \pm$ & $0.96 \pm$ & $0.02 \pm$ & $0.98 \pm$ & $0.05 \pm$ & $0.80 \pm$ & $0.23 \pm$ & $0.83 \pm$ & $1.0 \pm$ \\
\hline & & \pm 0.04 & 0.1 & 0.04 & 0.1 & 0.06 & 0.1 & 0.05 & 0.1 & 0.04 & 0.02 \\
\hline \multirow{2}{*}{$h_{s}$} & \multirow{2}{*}{$0.05^{*}$} & $0.04 \pm$ & $0.25 \pm$ & $0.04 \pm$ & $0.141 \pm$ & $0.05 \pm$ & $0.231 \pm$ & $0.03 \pm$ & $0.46 \pm$ & $0.01 \pm$ & $0.02 \pm$ \\
\hline & & 0.02 & 0.1 & 0.02 & 0.1 & 0.02 & 0.10 & 0.01 & 0.1 & 0.02 & 0.02 \\
\hline & $0.53 \pm$ & $0.47 \pm$ & $0.61 \pm$ & $0.41 \pm$ & $0.59 \pm$ & $0.35 \pm$ & $0.56 \pm$ & $0.40 \pm$ & $0.59 \pm$ & $0.45 \pm$ & $0.34 \pm$ \\
\hline & 0.1 & 0.03 & 0.04 & 0.03 & 0.04 & 0.04 & 0.04 & 0.04 & 0.04 & 0.03 & 0.05 \\
\hline
\end{tabular}




\begin{tabular}{|c|cc|cc|cc|cc|cc|c|}
$c$ & $0.89 \pm$ & $0.69 \pm$ & $0.89 \pm$ & $0.62 \pm$ & $0.87 \pm$ & $0.56 \pm$ & $0.84 \pm$ & $0.56 \pm$ & $0.89 \pm$ & $0.65 \pm$ & $0.5 \pm$ \\
& 0.1 & 0.03 & 0.04 & 0.07 & 0.04 & 0.08 & 0.04 & 0.07 & 0.04 & 0.06 & 0.03 \\
$\theta$ & $40^{\circ} \pm$ & & $40^{\circ} \pm$ & $37^{\circ} \pm$ & $40^{\circ} \pm$ & $40^{\circ} \pm$ & $40^{\circ} \pm$ & $38^{\circ} \pm$ & $40^{\circ} \pm$ & & \\
& $5^{\circ}$ & $38.3^{\circ} \#$ & $5^{\circ}$ & $5^{\circ}$ & $5^{\circ}$ & $3^{\circ}$ & $5^{\circ}$ & $6^{\circ}$ & $5^{\circ}$ & $38.3^{\circ} \#$ & $38.3^{\circ} \#$ \\
& $3.61 \mathrm{E}-$ & $5.33 \mathrm{E}-$ & $2.95 \mathrm{E}-$ & $1.53 \mathrm{E}-$ & $6.24 \mathrm{E}-$ & $9.30 \mathrm{E}-$ & $5.66 \mathrm{E}-$ & $2.67 \mathrm{E}-$ & $6.73 \mathrm{E}-$ & $5.31 \mathrm{E}-$ & $5.65 \mathrm{E}-$ \\
& 06 & 05 & 05 & 06 & 05 & 07 & 05 & 06 & 05 & 05 & 05 \\
\hline
\end{tabular}

* These values were set to the Domingue et al. (2002) Eros values due to the lack of opposition measurements in the Lederer et al. (2008) data sets. \# The value of the surface roughness was set to the average from the BVR results as this data set did not contain any measurements in the forward scattering ( $>90^{\circ}$ phase) region.

\# The value of the surface roughness was set to the average from the BVR results as this data set did not contain any measurements in the forward scattering $\left(>90^{\circ}\right.$ phase) region.

Table 4. Modern Hapke Model Parameter Values

\begin{tabular}{|c|c|c|c|}
\hline $\begin{array}{c}\text { Modern Hapke } \\
\text { Parameters }\end{array}$ & $\begin{array}{c}\text { B Filter } \\
(\mathbf{0 . 4 4} \boldsymbol{\mu} \mathbf{m})\end{array}$ & $\begin{array}{c}\text { V Filter } \\
\mathbf{( 0 . 5 4} \boldsymbol{\mu} \mathbf{m})\end{array}$ & $\begin{array}{c}\text { R Filter } \\
\mathbf{( 0 . 6 3} \boldsymbol{\mu} \mathbf{m})\end{array}$ \\
\hline$w$ & $0.54 \pm 0.03$ & $0.57 \pm 0.03$ & $0.64 \pm 0.03$ \\
\hline$B_{S O}$ & $0.96 \pm 0.11$ & $0.79 \pm 0.06$ & $0.07 \pm 0.03$ \\
\hline$h_{s}$ & $0.21 \pm 0.03$ & $0.15 \pm 0.06$ & $0.17 \pm 0.03$ \\
\hline$B_{C}$ & $0.35 \pm 0.02$ & $0.27 \pm 0.06$ & $0.51 \pm 0.02$ \\
\hline$h_{c}$ & $0.04 \pm 0.02$ & $0.04 \pm 0.02$ & $0.03 \pm 0.02$ \\
\hline$b$ & $0.42 \pm 0.02$ & $0.36 \pm 0.02$ & $0.43 \pm 0.02$ \\
\hline$c$ & $0.78 \pm 0.03$ & $0.65 \pm 0.04$ & $0.62 \pm 0.02$ \\
\hline$\theta$ & $40^{\circ} \pm 2^{\circ}$ & $40^{\circ} \pm 3^{\circ}$ & $40^{\circ} \pm 3^{\circ}$ \\
\hline $\mathrm{k}$ & $1.1 \pm 0.12$ & $1.1 \pm 0.1$ & $1.0 \pm 0.03$ \\
\hline$\chi$ & $6.54 \mathrm{e}-7$ & $3.79 \mathrm{e}-7$ & $1.02 \mathrm{e}-6$ \\
\hline
\end{tabular}



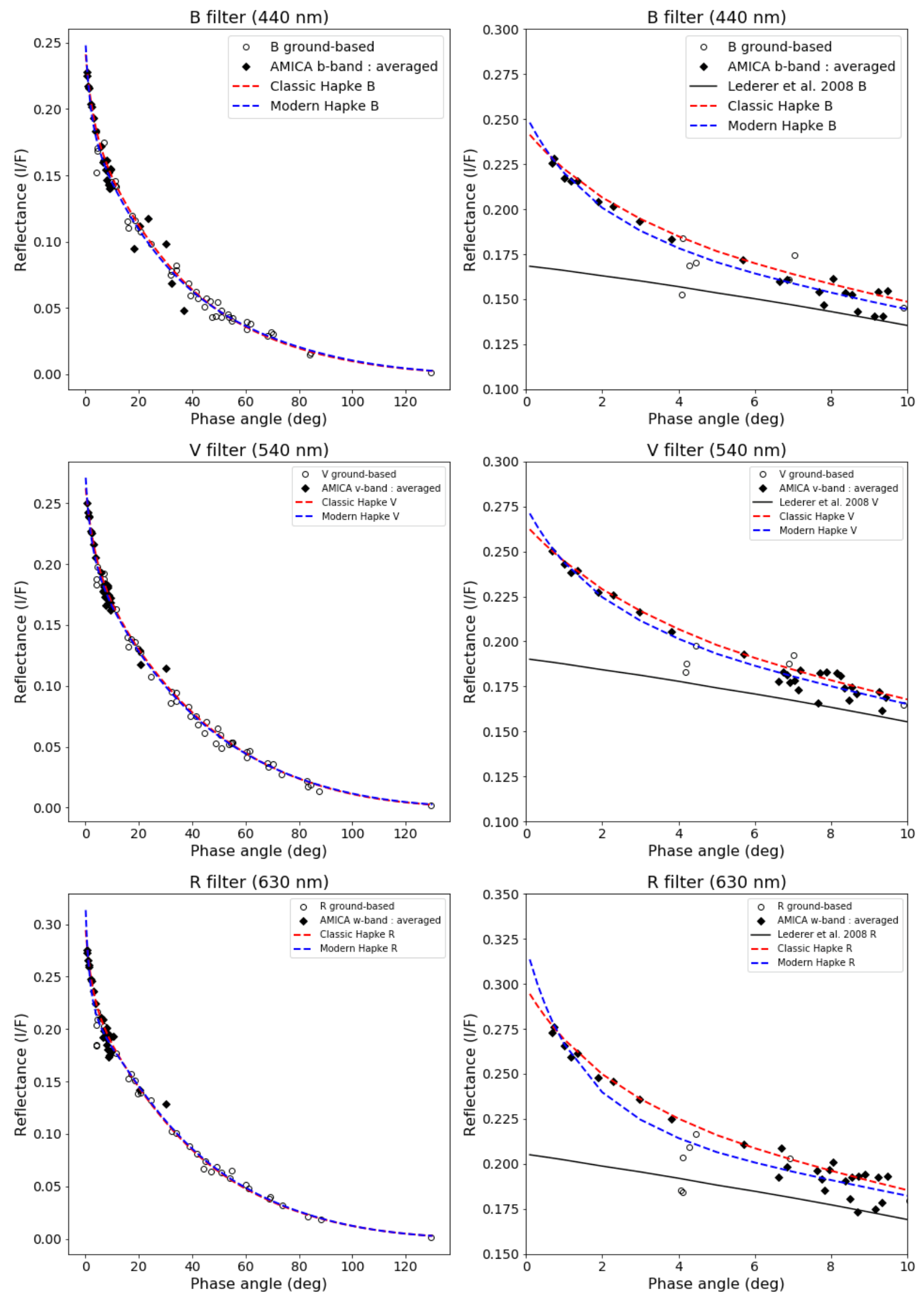
Figure 7. These graphs display the disk-integrated phase curves for Itokawa acquired in the $B(440 \mathrm{~nm}), V(540 \mathrm{~nm})$, and $R(630 \mathrm{~nm})$ filters. These phase curves contain both ground-based observations (Lederer et al. 2008), shown as open circles, and measurements extracted from the Hayabusa AMICA images, shown as grey diamonds. The Classic Hapke Model results from Lederer et al. (2008) are shown as a solid black line, the Classic Hapke Model results from this study are shown as a dashed red line, and the Modern Hapke Model results from this study are shown as dashed blue line. The left column graphs display the entire available phase curve, the right column graphs display the opposition region in each filter.
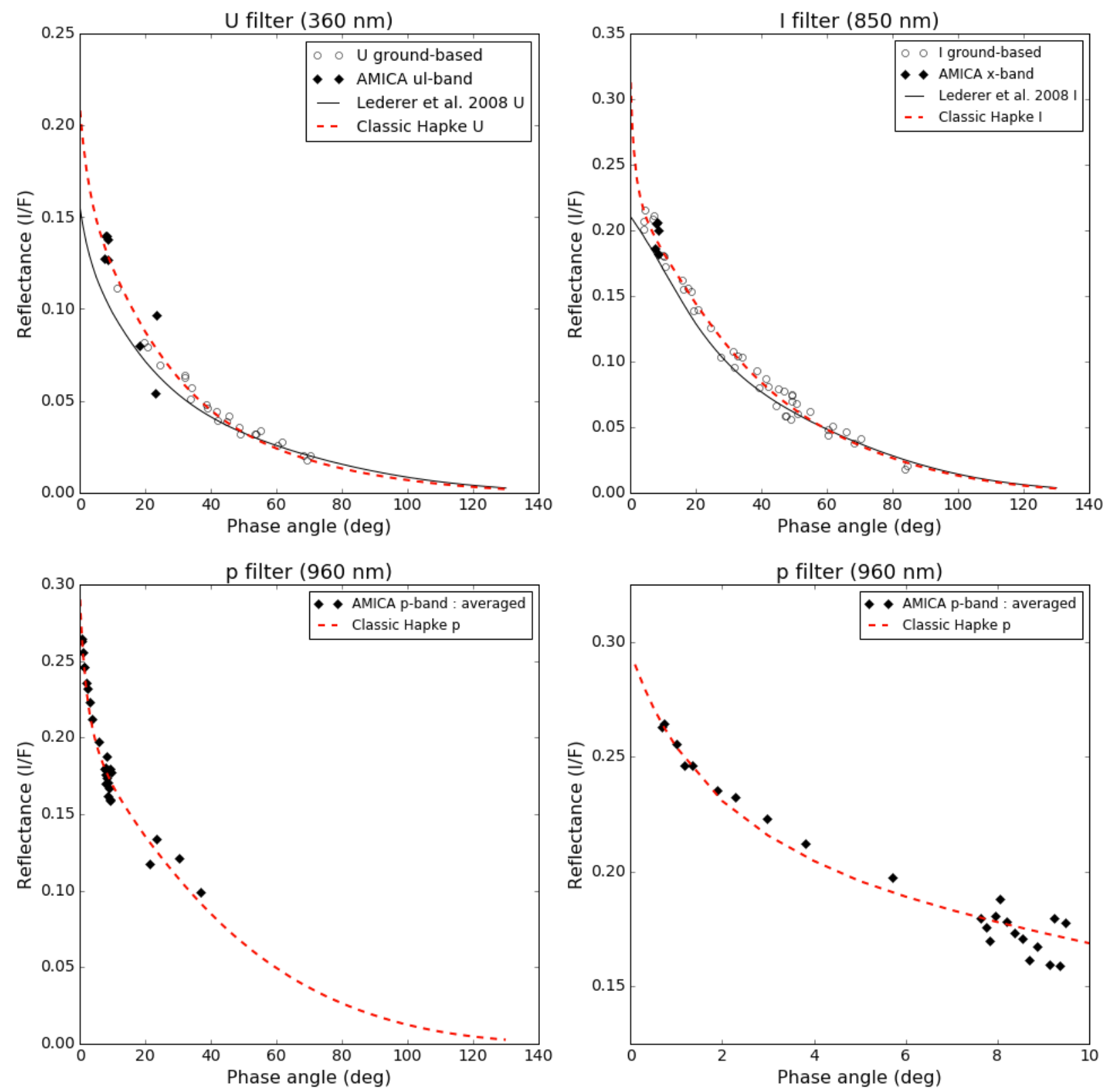

Figure 8. These graphs display the disk-integrated phase curves for Itokawa acquired in the $U(360 \mathrm{~nm}), I(850 \mathrm{~nm})$, and $p(960 \mathrm{~nm})$ filters. The U- and I- filter phase curves contain both 
ground-based observations (Lederer et al. 2008), open circles, and measurements extracted from the Hayabusa AMICA images, black diamonds. The p-filter phase curve is constructed solely from Haybusa AMICA image measurements. Each of these phase curves is modeled using the Classic Hapke Model. The Lederer et al. (2008) Classic Hapke Model is shown as a solid black line, the Classic Hapke Model results from this study are shown as a dashed red line. The opposition regions for the AMICA p-band data are shown in more detail in the bottom right graph. Insufficient phase angle coverage prohibited application of the Modern Hapke Model to these data sets.

A quick examination of the results shows that the SSA values mimic the spectral shape of the reflectance data (Figure 9), as expected from the modeling constraints. Surface roughness parameters from the two Classic Hapke models and the Modern Hapke model all display a rough surface, with values near $40^{\circ}$. This is commensurate with the gravel-like regolith observed in the AMICA images (Figure 1 (a)).
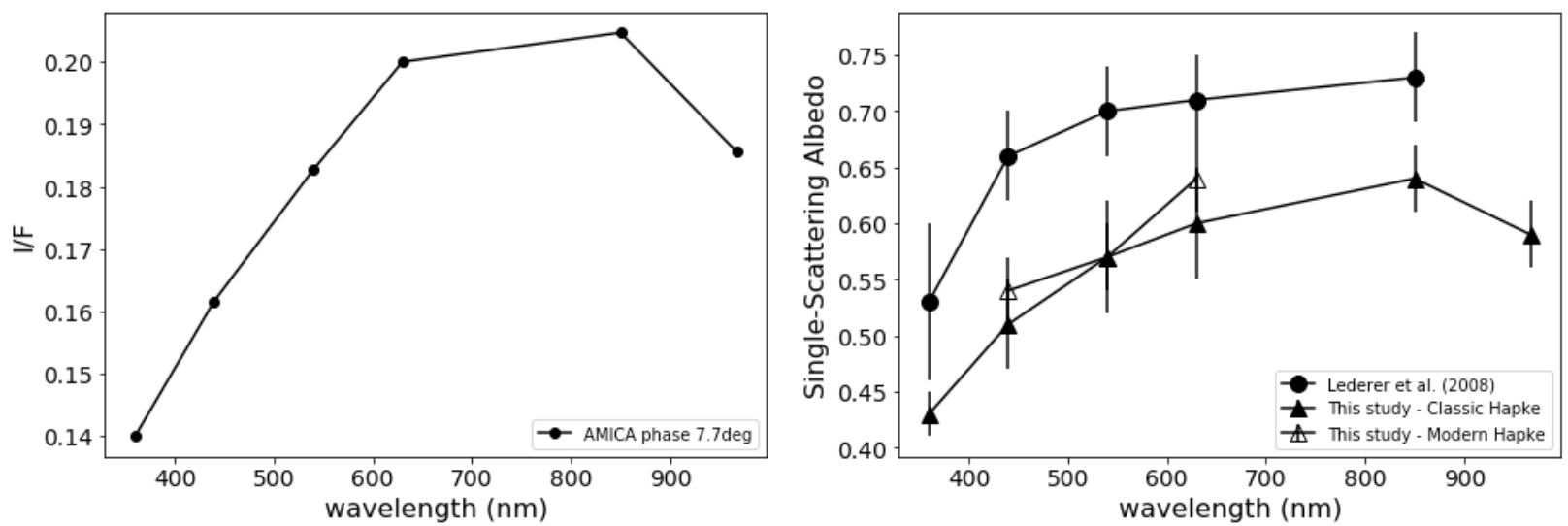

Figure 9. These graphs compare the spectral shape of the Itokawa reflectance spectrum (left) acquired at $7.7^{\circ}$ phase with the spectral shape of the SSA spectrum (right) from the modeling results of Lederer et al. (2008), black circles, the Classic Hapke model from this study, black triangles, and the Modern Hapke model from this study, open triangles.

\subsection{Validation of the Classic Hapke Model}

The simulated images can be synthesized using the disk-resolved Hapke model equations with the Hapke parameters derived from this study and geometry information from the mission SPICE kernels. Here we compare the simulated images based on the Classic Hapke Model and the actual observed images. Figure 10 shows the reflectance ratio between the synthesized images and the observed images at two different phase angles, $8.1^{\circ}$ and $30.3^{\circ}$. The two right columns display the illumination (incidence angle) and viewing (emission angle) geometry used for the simulated images based on the modeling results. The same model parameters are used over the entire surface, although variations in the parameter values are expected based on the albedo variations seen in the AMICA imagery. 
Figure 11 shows the average of the reflectance ratio with the error of standard deviation in each $10^{\circ}$ bin. Areas imaged at large incidence or emission angle tend to have under-estimated values in reflectance. This trend was also observed in the Mercury imaging data acquired by the MErcury Surface, Space ENvironment, GEochemistry, and Ranging (MESSENGER) mission (Domingue et al. 2016). We note that for example at phase angle $30.3^{\circ}$, the estimation from the Classic Hapke Model slightly overestimates the reflectance even for small incidence and emission angle $\left(i<30^{\circ}\right.$ and $\left.e<30^{\circ}\right)$. This suggests that the Classic Hapke Model may not sufficiently predict the reflectance of the areas observed at large phase angles $>30^{\circ}$. However, for example at phase angle $8.1^{\circ}$, the Classic Hapke Model well describes the surface of Itokawa within the error of $10 \%$ for $i<50^{\circ}$ and $e<50^{\circ}$. It should be noted that the photometry correction based on the model derived from disk-integrated observations may cause artificial color differences along the edge of an object where incidence and emission angles are large.

(a)
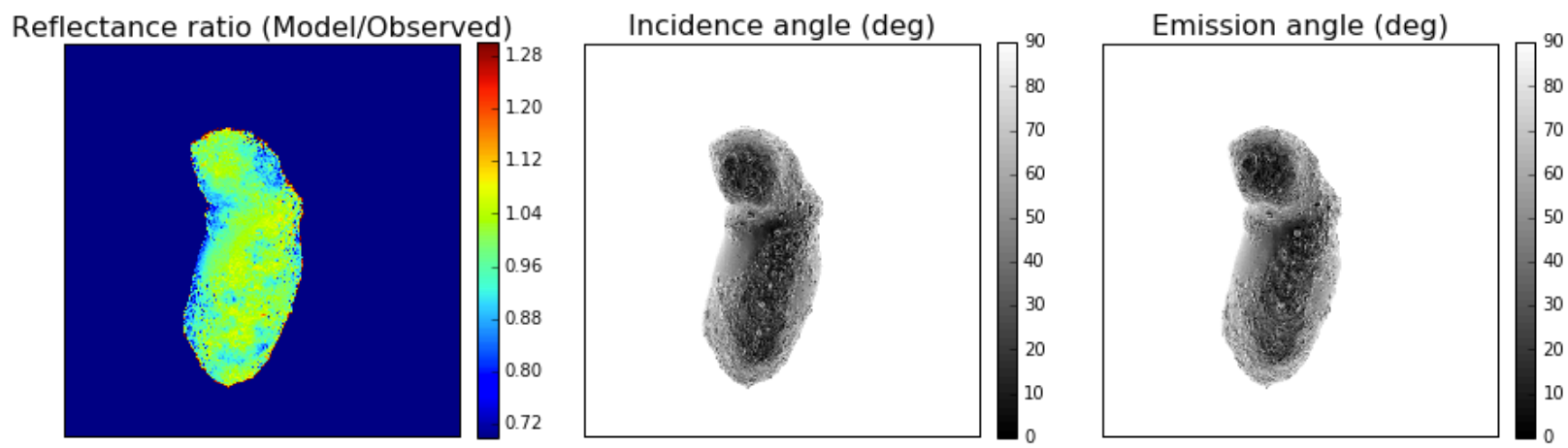

(b)
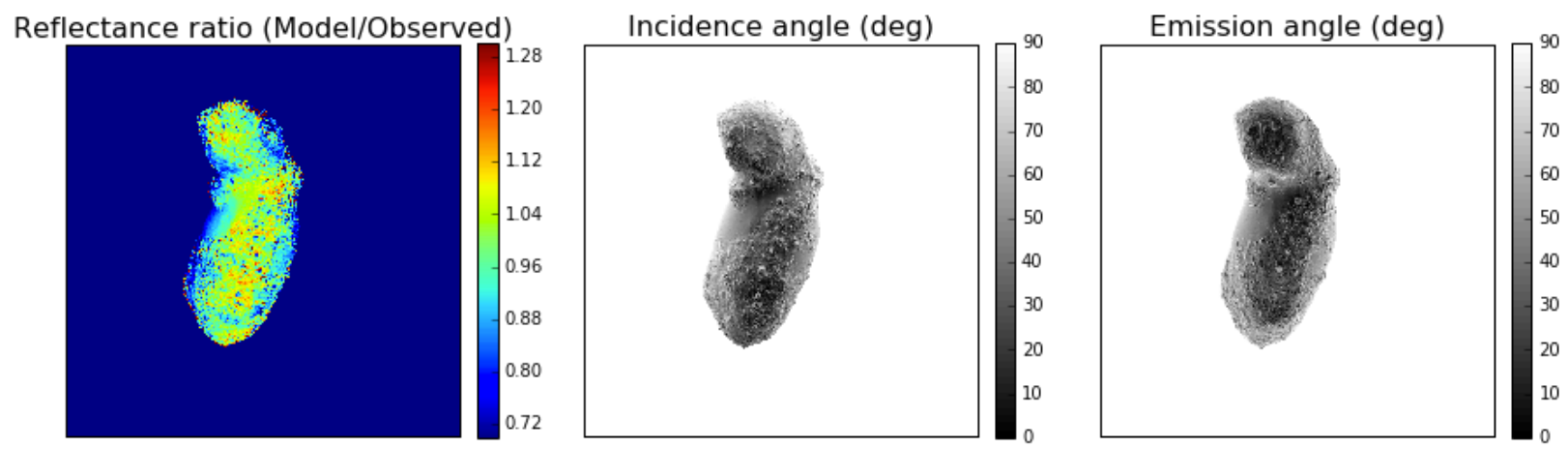

Figure 10. Comparison between the model reflectance and the observed reflectance:

$S T \_2385578902 \_v$ at phase angle $8.1^{\circ}(a)$ and $S T_{-} 2442680854 \_v$ at phase angle $30.3^{\circ}(\mathrm{b})$. The areas where the reflectance is underestimated corresponds to places imaged at larger $\left(>50^{\circ}\right)$ incidence and emission angle. 

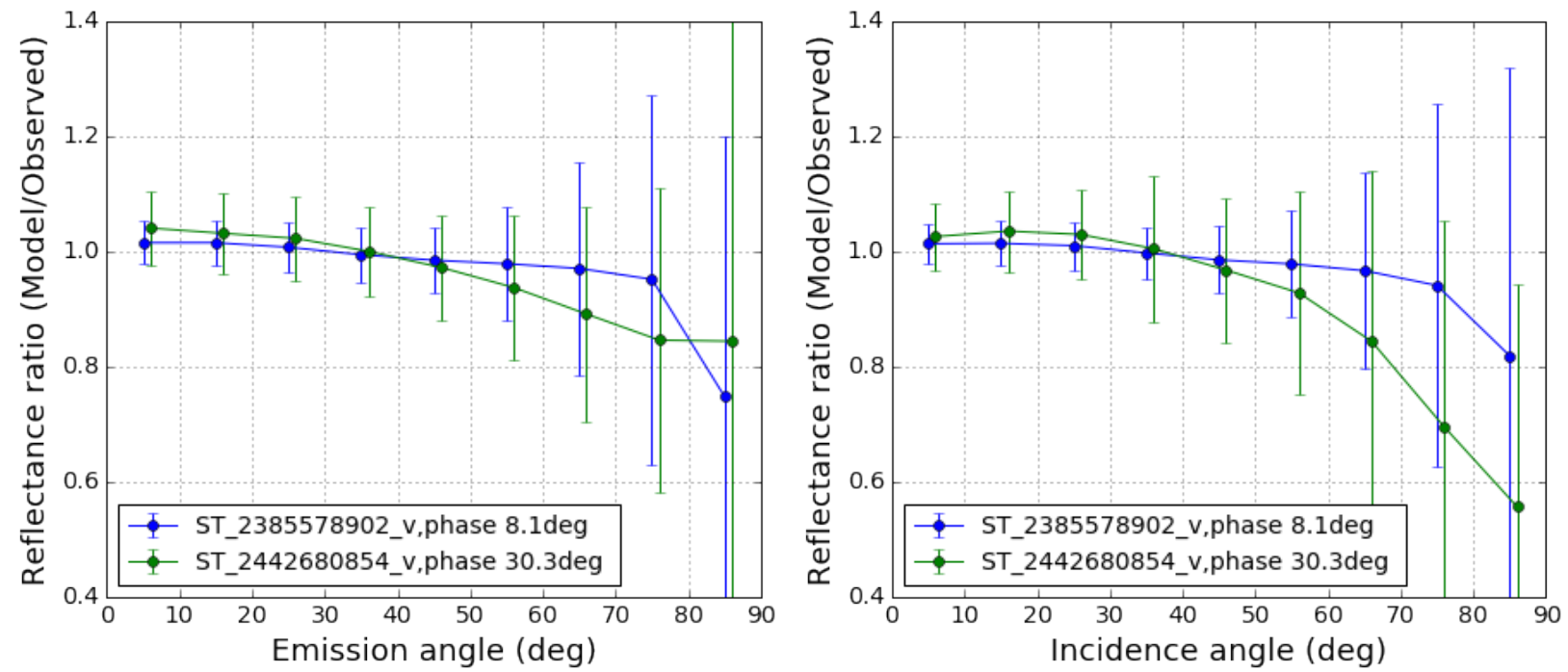

Figure 11. The reflectance ratios for both images in Figure 10 are shown as functions of emission (left) and incidence (right) angles. The reflectance at small phase angle $\left(8.1^{\circ}\right)$ is well predicted within the error of $10 \%$ by the Classic Hapke Model for $i<50^{\circ}$ and $e<50^{\circ}$. However, the reflectance across the surface imaged at a larger angle $\left(30.3^{\circ}\right)$ shows that the places imaged at large incidence and emission angles underestimate and those imaged at small incidence and emission angles slightly over estimate the observed reflectance.

\subsection{Geometric and Bond albedos}

The geometric albedo, $p$, is the reflectance seen at zero phase angle relative to a Lambertian surface of the same cross-sectional area similarly illuminated. This can be calculated directly from the Hapke equations (e.g. Hapke 1984, 2012a).

The phase integral, $q$, and Bond albedo, $A_{B}$, are related to the geometric albedo, $A_{p}$, by the relation:

$$
A_{B}=A_{p} q \quad \operatorname{Eq}(4.1)
$$

The phase integral is calculated from the definition:

$$
q=2 \int_{0}^{\pi} \phi(\alpha) \sin \alpha d \alpha \operatorname{Eq}(4.2)
$$

where $\phi(\alpha)$ is the reflectance. Using the Romberg method of integration, the phase integral for each filter was calculated using both the Classic and Modern Hapke models. The Classic Hapke model results are compared with the Lederer et al. (2008) values in Table 5. The Geometric albedo derived from the Modern Hapke modeling results for the BVR filters are $0.26 \pm 0.02,0.27 \pm 0.02$, and $0.32 \pm 0.02$, respectively, and are equivalent to the Classic Hapke Model values within the error bars.

Table 5. Albedos and Phase Integral - Classic Hapke Model

\begin{tabular}{|c|c|c|c|} 
Filter & Geometric Albedo & Phase Integral & Bond Albedo
\end{tabular}




\begin{tabular}{|c|c|c|c|c|c|c|}
\hline & $\begin{array}{c}\text { Lederer et } \\
\text { al. (2008) }\end{array}$ & $\begin{array}{c}\text { This } \\
\text { study }\end{array}$ & $\begin{array}{c}\text { Lederer et } \\
\text { al. (2008) }\end{array}$ & $\begin{array}{c}\text { This } \\
\text { study }\end{array}$ & $\begin{array}{c}\text { Lederer et } \\
\text { al. (2008) }\end{array}$ & This study \\
\hline U & $0.15 \pm 0.05$ & $0.21 \pm 0.05$ & $0.08 \pm 0.02$ & $0.08 \pm 0.02$ & $0.012 \pm 0.01$ & $0.016 \pm 0.02$ \\
\hline B & $0.17 \pm 0.02$ & $0.24 \pm 0.02$ & $0.09 \pm 0.01$ & $0.11 \pm 0.02$ & $0.0153 \pm 0.01$ & $0.026 \pm 0.02$ \\
\hline V & $0.19 \pm 0.02$ & $0.27 \pm 0.02$ & $0.11 \pm 0.01$ & $0.13 \pm 0.02$ & $0.0209 \pm 0.01$ & $0.033 \pm 0.02$ \\
\hline R & $0.21 \pm 0.02$ & $0.30 \pm 0.02$ & $0.12 \pm 0.01$ & $0.14 \pm 0.02$ & $0.0252 \pm 0.01$ & $0.041 \pm 0.02$ \\
\hline I & $0.21 \pm 0.02$ & $0.32 \pm 0.02$ & $0.12 \pm 0.01$ & $0.14 \pm 0.02$ & $0.0252 \pm 0.01$ & $0.044 \pm 0.02$ \\
\hline p & n/a & $0.30 \pm 0.02$ & n/a & $0.14 \pm 0.02$ & n/a & $0.040 \pm 0.02$ \\
\hline
\end{tabular}

\subsection{Discussion}

\subsection{Surface Regolith Properties Implied by the Opposition Effect}

The addition of the AMICA data has provided new, additional information in the opposition regime of Itokawa's phase curve over multiple wavelengths. Itokawa's phase curve contains a more narrow opposition peak than previously observed. The Classic Hapke Model assumes that the opposition effect is due entirely to SHOE, while the Modern Model accounts for both SHOE and CBOE components. In this section, we discuss the physical properties implied by the model opposition parameters from both models. These properties are compared with the radar observations and sample analyses to establish any consistency between the various measurements.

Both the Classic and Modern Hapke models were derived from the radiative transfer equations, with additions and modifications based on the physical properties particular to particulate medium. For the complete derivation of these equations, especially the theoretical correlations between model parameters to surface physical properties, the reader is referred to Hapke (2012a) and the references therein. While applications of these models to laboratory data have shown some qualitative correlations between laboratory sample properties and model parameters (Shepard and Helfenstein 2007, Helfenstein and Shepard 2011, and Souchon et al. 2011), quantitative correlations remain elusive. It still remains productive to compare the predicted surface properties based on the model parameters to those derived from the high-spatial resolution images, radar observations, and sample analyses to ascertain the viability of these models to both meet early mission planning goals and understand surfaces across the solar system.

One purpose of this study is to examine the predictive capability of these models in the early mission stages, where image data do not have sufficient spatial resolution, to provide insight on regolith properties for landing and/or sample collection preparation. The approach for determining the predictive capability was to relate the model parameter values to the surface based on the original definitions from the model's mathematical derivation. These predicted properties are then compared to the surface properties as seen in the higher spatial resolution images acquired later in the mission and the particle properties seen in the returned samples. While the samples were acquired in one region and may not represent the entire surface, they do provide an initial set of constraints. Through these comparisons we can meet the second goal of the paper: a better understanding of Itokawa's surface. 


\subsubsection{Transport Mean Free Path from the Modern Hapke Model}

The width of the CBOE portion of the opposition effect is wavelength dependent, such that

$$
h_{c}=\frac{\lambda}{4 \pi \Lambda_{T}}, \quad \operatorname{Eq}(5.1)
$$

where $\lambda$ is the observational wavelength and $\Lambda_{T}$ is the transport mean free path (from Hapke 2012a, eq. 9.41b). Some constraints regarding grain structures can be derived using this relationship.

Using the Modern Hapke modeling results, the transport mean free path within the regolith was calculated, and is given in Table 6. The transport mean free path is defined as the mean distance a photon will travel before encountering a major scattering center. A major scattering center changes a photon's direction of motion by a significant amount $\left(>90^{\circ}\right)$. The transport mean free path varies as a function of wavelength, placing a constraint on the size and potential distribution of scattering centers. The modeling results predict a photon will travel about twice its length in distance prior to being significantly scattered. Table 11 shows the range of values for the transport mean free path over the wavelength range of the photometric data currently available.

Table 6. Transport Mean Free Path

\begin{tabular}{|c|c|c|}
\hline$\lambda(\mu \mathrm{m})$ & $\mathrm{h}_{\mathrm{c}}$ & $\Lambda_{\mathrm{T}}(\mu \mathrm{m})$ \\
\hline 0.44 & 0.04 & 0.88 \\
\hline 0.54 & 0.04 & 1.08 \\
\hline 0.63 & 0.03 & 1.68 \\
\hline
\end{tabular}

The samples returned from Itokawa by the Hayabusa spacecraft provide some insight into the structure of regolith grains that can be compared with these photometry results. One must keep in mind that the photometry results presented here are a depiction of an idealized, global mean surface and the samples are representative of a single geologic unit on Itokawa. However, it is still useful to make the comparison to determine if the two sources of information on regolith grain structure are consistent. Images of the Hayabusa sample site in Muses Sea show a regolith with grain sizes ranging from millimeters to centimeters (Yano et al. 2006). The particles collected from the sample sites are a few 100 $\mu \mathrm{m}$ in diameter (Tsuchiyama et al. 2011). The returned particles show substantial textural variations, including cracks, inclusions, and multiple mineral phases (Tsuchiyama et al. 2011, Mikouchi et al. 2014, Nakamura et al. 2011, 2014). Many of the samples show sharp grain boundaries between minerals (Mikouchi et al. 2014, Nakamura et al. 2014) while some appear to be agglomerates of silicate grains of $5 \mu \mathrm{m}$ or smaller with many interstitial pores (Nakamura et al., 2014). Inclusions of $3-10 \mu \mathrm{m}$ are observed in some of the samples (Nakamura et al. 2011, 2014), along with elongated pores that are assumed to be the result of annealing of impact-generated fractures (Nakamura et al. 2011, 2014). The particles, for 
the most part, show good crystallinity (Mikouchi et al. 2014, Nakamura et al. 2014). The structures seen in the samples (cracks, inclusions, pores) all have the potential to serve as major scattering centers on micrometer or larger scales. Some (sub-)micron-sized structures are also found within the Itokawa particles. For example, submicron-sized craters are found on several returned samples, which may have been created by the impact of high-speed secondary nanoparticles produced by an impact into the regolith (Nakamura et al., 2011; Harries et al., 2016). Harries et al. (2016) counted 15 craters ranging $200-500$ $\mathrm{nm}$ on the sample RA-QD02-0265. The mean distance of these craters was $6.21-8.79 \mu \mathrm{m}$. As will be shown in section 5.4, the photometric model predicts single-particle phase function that contains both a forward and a backward scattering component. The submicron physical structures, such as the craters, will contribute to the backward scattering component while the phase changes (such as amorphous to crystalline, or mineral to void or vesicles) will contribute to the forward scattering component. The frequency of a photon encountering such a scattering center (approximately once every micron) suggests that those structures associated with forward scattering have a higher probability of being encountered than those associated with backward scattering. The microscopic physical properties seen within the returned samples are consistent with the properties implied by the transport mean free path derived from the model.

Examination of a subset of the particles for space weathering effects shows that the structure of the grains can be described by a series of rims, layers, or zones (Noguchi et al. 2013). The top rim (Zone I) is amorphous and ranges in depth from $2-3 \mathrm{~nm}$, the intermediate rim (Zone II) is partially amorphous with localized crystalline structures ranging from $30-80 \mathrm{~nm}$ in size, and the bottom layer (Zone III) is the crystalline substrate of the grain (Noguchi et al. 2013). Zone I and Zone II both can contain nanophase Fe, and the composite vesicular rim also contains vesicles. The mode size of the nanophase Fe is 1.5 $-2 \mathrm{~nm}$ while the vesicles show an oval shape that's $\sim 20 \mathrm{~nm}$ normal to the exterior surface and $\sim 50 \mathrm{~nm}$ parallel to the exterior surface (Noguchi et al. 2013).

The wavelength of the reflectance measurements studied here are 440 to $630 \mathrm{~nm}$, a hundred times larger than the space weathering structures seen in the returned samples. The transport mean free paths over this wavelength range span between 880 to $1681 \mathrm{~nm}$, indicating that the grain structures, such as rims and nanophase Fe, due to space weathering are not influencing the scattering properties at these wavelengths.

\subsubsection{Porosity from the Modern Hapke Model}

The Modern Hapke Model also includes an explicit porosity-related parameter, $k$. This parameter $k$ is related to the porosity by the expression

$$
k=\frac{-\ln \left(1-1.209 \phi^{\frac{2}{3}}\right)}{1-1.209 \phi^{\frac{2}{3}}}, \quad E q(5.2)
$$

Hapke (2012a), where $\phi$ is the filling factor (related to porosity $(\rho)$ by $\rho=1-\phi)$. The Modern Hapke model results for the BVR data sets show a narrow range of values for $k$ 
from 1 - 1.1, which correspond to porosity values between $77 \%$ and $79 \%$. This value is much higher than the bulk porosity of Itokawa $40 \%$ (Saito et al., 2006). This is not very surprising since photometry is sensitive to the topmost, optically active layer of the surface (a few micrometers of depth) and describes the properties of just this topmost layer. The radar observations by Ostro et al. (2004) estimated the surface bulk density of Itokawa to be $1.66_{-0.65}^{+0.35} \mathrm{~g} / \mathrm{cm}^{3}$, based on the method by Magri et al. (2001). Considering a density of the average returned samples of $3.4 \mathrm{~g} / \mathrm{cm}^{3}$ (Tsuchiyama et al., 2011), this suggests a surface porosity in the range of $41 \%-70 \%$. The surface porosity $77 \%-79 \%$ derived from the Modern Hapke Model here is slightly higher than this radar derived porosity. This may be due to the depth to which each technique is sensitive. Our surface porosity reflects nm-to- $\mu \mathrm{m}$ scaled information, while the radar observation describes deeper cm-to-m scaled surface properties. Our photometry result indicates that the surface of Itokawa is very fluffy at the depths of several microns. Radar and bulk porosities further indicate the asteroid regolith becomes more compact with depth.

\subsubsection{Porosity from the Classic Hapke Model}

Because of the larger phase angle coverage for BVR filters, the modeling results for these data sets are better constrained than the other wavelengths examined. Therefore, we focus the remainder of our discussion of the opposition region to these three filters.

The width of the SHOE component can be correlated to grain size distribution and porosity via the relationship

$$
h_{s}=-\left(\frac{3}{8}\right) Y \ln (\rho), \quad E q(5.3)
$$

where $\rho$ is the porosity and $Y$ is the grain size distribution function which is related to the power law index $\beta$ of the differential size distribution of grains, i.e., $d N / d r \propto r^{-\beta}$ (Hapke 1986, 2012a). The grain size distribution functions are shown in Table 7, where ${ }^{r} / r_{s}$ is the ratio of the cut-off radius of the largest grain to the smallest grain size. Once we establish a size distribution function for Itokawa's regolith, we can then estimate the regolith porosity from the Hapke parameter $h_{s}$.

Several studies of boulder counting based on the AMICA images displayed a low power law distribution for Itokawa, with $\beta$ constrained to range between $\sim 2.7$ to $\sim 4.2$. (Saito et al., 2006; Michikami et al., 2008; Mazrouei et al., 2014; Tancredi et al. 2015). Tancredi et al. (2015) pointed out that the differential power law index, $\beta$, may be smaller for small grains. Examination and investigation of the size distribution within the returned samples by Tsuchiyama et al. (2011) suggests that the samples from smooth terrain within Muses Sea (from which the samples were acquired) can be represented with a differential power law index between 3 to 3.8. Thus, it is reasonable that we assume $\beta=3-4$ for Itokawa in our analyses.

The boulder counts by Tancredi et al. (2015) show size ranges from $19 \mathrm{~cm}$ to $\sim 30 \mathrm{~m}$ in diameter. The $r_{l} / r_{s}$ value using just the Tancredi et al. (2015) boulder size range is 157.9. Note that there may be a detection limitation of grain size due to the pixel resolution of image. Surficial features that appear to be solid boulders, rocks, or pebbles 
could be clumps of much finer unresolved material. Thus, the ratio $r_{l} / r_{s}$ would be larger. Figure 12 is calculated based on the relationship between the width of SHOE $h_{s}$ and porosity from eq. (5.3). This suggests that the $h_{s}$ values from the Classic Hapke model are in good agreement with the porosity which was derived from the k parameter, although neither strongly constrains the porosity.

Table 7. Grain Size Distribution Functions

\begin{tabular}{|c|c|}
\hline $\boldsymbol{\beta}$ & $\boldsymbol{Y}$ \\
\hline 1 & $3 / \sqrt{8 \ln \left(r_{l} / r_{s}\right)}$ \\
\hline 2 & $2 \sqrt{r_{s}} / r_{l}$ \\
\hline 3 & $\sqrt{2}\left[\ln \left(r_{l} / r_{s}\right)\right]^{1.5}\left(r_{s} / r_{l}\right)$ \\
\hline 4 & $\sqrt{3} / \ln \left(r_{l} / r_{s}\right)$ \\
\hline
\end{tabular}

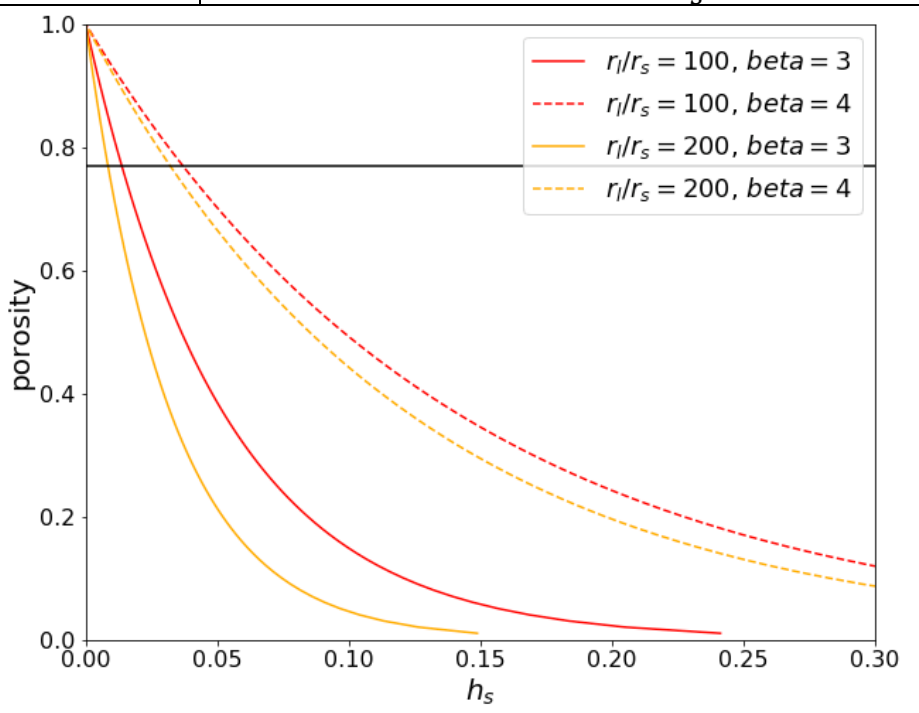

Figure 12. The relationship between the width of SHOE $h_{s}$ and porosity from eq. (5.3). We chose realistic parameters for Itokawa, $\beta=3-4, r_{l} / r_{s}=100-200$. The black line shows the porosity 0.77 from the Modern Hapke Model porosity parameter $k$.

\subsubsection{Cohesive force on Itokawa}

The constraints on the cohesive strength of the regolith are important for estimating crater size, regolith mobility, and surface rejuvenation. Constraints on the cohesiveness of the surface are also important to understand in the early stages of planning a landing on, or sample collection from, the regolith. Cohesive forces, especially the van der Waals force, play a more important role for smaller bodies, due to their small gravity potential, than on larger bodies (Scheeres et al., 2010). The cohesive strength for Itokawa's regolith can be estimated based on the porosity derived from the Modern Hapke Model. Assuming that Itokawa's surface regolith is composed of granular material with no internal 
micro-porosity, we can compute the bond number, $\mathrm{B}$, which is defined as the ratio of the gravity and inter-particle forces. Yu et al. (2003) provided an empirical relationship between the porosity $\rho$ and the bond number $B$, such that

$$
\rho=\rho_{0}+\left(1-\rho_{0}\right) \exp \left(-m B^{-n}\right), \quad E q(5.4)
$$

where $\rho_{0}, m$, and $n$ are constants. We used the $\rho_{0}=0.36, m=1.821$, and $n=0.158$ values from Kiuchi and Nakamura (2014). Note that, this relationship works for material with no internal micro-porosity. That is, the particles themselves are not porous. Our photometrically derived porosity $\rho=0.77-0.79$ leads to high Bond number values between 6000 - 12000. This suggests that the cohesive force is more than a thousand times stronger than the gravity force on Itokawa.

As seen in the images, Itokawa is covered mostly by coarse gravels, ranging from $\mathrm{cm}$ to $\mathrm{m}$ diameter in scale (Tancredi et al. 2015). Coarse grains of this size scale typically correspond to low bond numbers. For example, Scheeres et al. (2010) showed that a 0.2-m gravel corresponds to a bond number of 1 on Itokawa. The high bond number derived from the photometric porosity may indicate that the assumption of no internal micro-porosity may be incorrect. Or these bond number values may indicate that our understanding of the optically active portion of the regolith (that portion of the regolith measured in the photometric data, typically on the order of $\mu \mathrm{m}$ to $\mathrm{mm}$ ) is incomplete.

The bond numbers suggested by our photometrically-derived porosity assumes particle sizes between $\mathrm{mm}$ to $\mathrm{cm}$ in diameter, equivalent to those observed in the imaging data for Muses Sea (Yano et al. 2006). However, there is a possibility that very fine inter-particle grains bond the large gravels together. Given that the reflectance measurements sample only the optically active layer (on the order of $\mu \mathrm{m}$ to $\mathrm{mm}$ ), it is highly probable that the photometrically-derived porosity is mostly related to the fine-grained regolith component which may be either bonding the larger particles or covering the coarser grained materials. For example, Sanchez and Sheeres (2014) suggested the possible bonding strength of finer grain between large particles. The size of the samples returned by Hayabusa attest to the existence of such small ( $\mu \mathrm{m}$ or less) material on Itokawa's surface. Thus, the bond numbers derived from the photometric porosity indicates that the inter-particle forces may play a much larger role than gravity in maintaining a fine-grained component in the top mm or so of regolith on Itokawa, and that component may have an internal micro-porosity.

\subsection{Surface roughness}

The AMICA images show that the grain size distribution across the surface of Itokawa is highly variable (Figure 1). Examination of regolith migration and sorting using high-resolution images of Itokawa's surface demonstrate that the regolith is comprised of unconsolidated millimeter-sized and larger gravels (Miyamoto et al., 2007; Tancredi et al., 2015). Though Itokawa's overall structure has an impact-generated origin, it's surface regolith has been further modified by processes including granular convection, landslide-like granular migrations, and particle sorting, which have resulted in the segregation of the regolith into areas with fine grains in regions of gravitational potential lows, such as Sagamihara region and Muses Sea (Miyamoto et al. 2007; Tancredi et al., 
2015). Yano et al. (2006) examined the Hayabusa landing site observations and concluded that, at the landing site, the regolith was comprised of size-sorted, mm-to-cm-sized grains. This is in comparison to the regolith elsewhere on the surface that could be described with sub-centimeter to meter-sized gravel (Yano et al. 2006). However, it should be noted that due to the limitation of pixel resolution of the images, finer regolith $<1 \mathrm{~mm}$ may exist on its surface. The returned samples are much smaller than $1 \mathrm{~mm}$ (Tsuchiyama et al., 2011).

The observations examined in this study, however, are disk-integrated and represent Itokawa's regolith properties on a globally averaged scale. It is of interest to examine how the modeling results describe the global surface regolith properties. The roughness parameter $\theta$ models the average slope within each unresolved surface patch. We obtained values of $\theta$ ranging from $37^{\circ}-40^{\circ}$. That is, the disk-integrated photometry cannot be independent of the global shape of the object. For example, Domingue et al. (2002) derived $\theta=36^{\circ} \pm 5^{\circ}$ from the disk-integrated photometry of Eros; this is larger than from disk-resolved photometry by Li et al. (2004), with a value of $\theta=28^{\circ} \pm 3^{\circ}$. We have taken into account the detailed shape of Itokawa in this study. The effect of global shape must be much smaller than Domingue et al. (2002) which assumes a spherical shape for Eros. It may be reasonable to compare with the value from Li et al. (2004), which is smaller than that of Itokawa from this study. This is consistent with the close-up images from both spacecraft. However, it should be noted that the roughness parameter derived by the NIRS disk-resolved photometry is smaller than ours. This might suggest the irregular global shape of Itokawa affects the average roughness derived here. Again, in this study we can only discuss the disk-average property, and it is not straight forward to interpret the roughness parameter. We will conduct local photometry analysis on the comparison of the roughness parameter and topological feature in a future, follow-on study.

\subsection{Single Scattering albedo (SSA)}

The single scattering albedo (SSA or $\mathrm{w}$ ) is defined as $w=\frac{S}{S+K}$, where $\mathrm{S}$ is the scattering coefficient and $K^{\prime}$ is absorption coefficient. Itokawa has a slightly higher SSA value $(0.57 \pm 0.05)$ in the visible wavelength range compared with Eros $(0.43 \pm 0.02)$, suggesting scattering and not absorption plays a larger role in the interaction of the surface with incident sunlight. The high SSA is theoretically explained by both composition and the grain size in Mie scattering (Hapke, 1993). Thus, the smaller grain medium has the larger value. However, the surface of Itokawa is observed to be mostly covered by coarse grains with a gravel-like texture. Moreover, some experiments suggest that the surface roughness and the porosity have a negative correlation with the SSA (Kaasalainen, 2003; Shepard and Helfenstein, 2007). However, our results cannot be explained by these suggested correlations, as this would suggest Itokawa's regolith is comprised of smaller grains, is less rough, and less porous than Eros' surface (Domingue et al., 2002). There are two additional possibilities to account for this difference: 1) the higher SSA for Itokawa compared to Eros suggests that the coarse grains observed in the Itokawa images are covered with a thin layer of fine particles, some of which were collected by Hayabusa's sample collector, or 2) the higher SSA for Itokawa compared to Eros corresponds to the composition and alteration of material itself. Space weathering on S-type asteroids is a well-known process that darkens surface material (Sasaki et al., 1999). Thus, the degree of space weathering on 
Itokawa may be weaker than that on Eros. This is supported by the young surface age for Itokawa, as estimated from the crater age (Tatsumi and Sugita, 2018), the spectral ages of the returned samples (Bonal et al., 2015), and the remote-sensing observations (Koga et al., 2018).

\subsection{Single Particle Scattering Behavior}

The two parameters of the Henyey-Greenstein phase functions derived from both the Classic and Modern Hapke modeling results from this study are less forward scattering than those predicted by Lederer et al. (2008) (Figure 13). Both the amplitude parameter b and the partition parameter $\mathrm{c}$ are smaller than those derived by Lederer et al. (2008) and are similar between the Classic and Modern Hapke modeling results (Figure 14). There is no distinctive wavelength dependence on the particle scattering behavior (Figures 14 \& 15). Note that these scattering properties are on the microscopic level, interacting with structures within the regolith grains.

The resulting $b$ and $c$ parameters imply that the scattering centers present within the regolith grains have a higher probability of scattering light in the forward direction. This may be due to either: 1) the phase curves contain insufficient coverage to fully characterize the forward direction (the phase angle coverage does not go beyond $\sim 130^{\circ}$ ), 2) the scattering centers having similar probabilities for directing light in both the forward or backward scattering direction, or 3) a combination of these two sources. Scattering centers include such items as cracks, bubbles, inclusion, or mineral phase changes. The spectral behavior of the transport mean free path suggests that an additional property for these scattering centers: they are on the order of 880 to $1681 \mathrm{~nm}$ apart (Section 5.1.1). 
U filter $(360 \mathrm{~nm})$

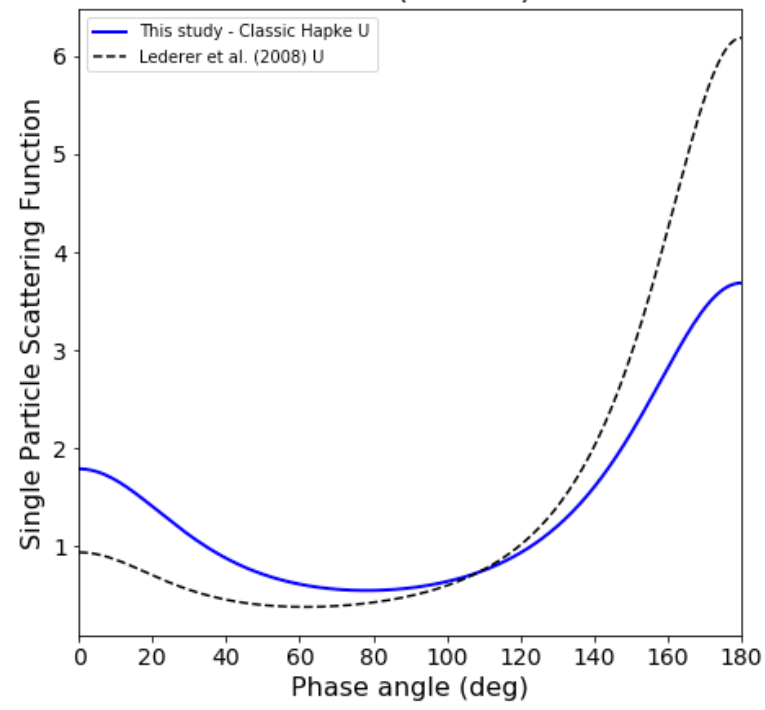

$\mathrm{V}$ filter $(540 \mathrm{~nm})$

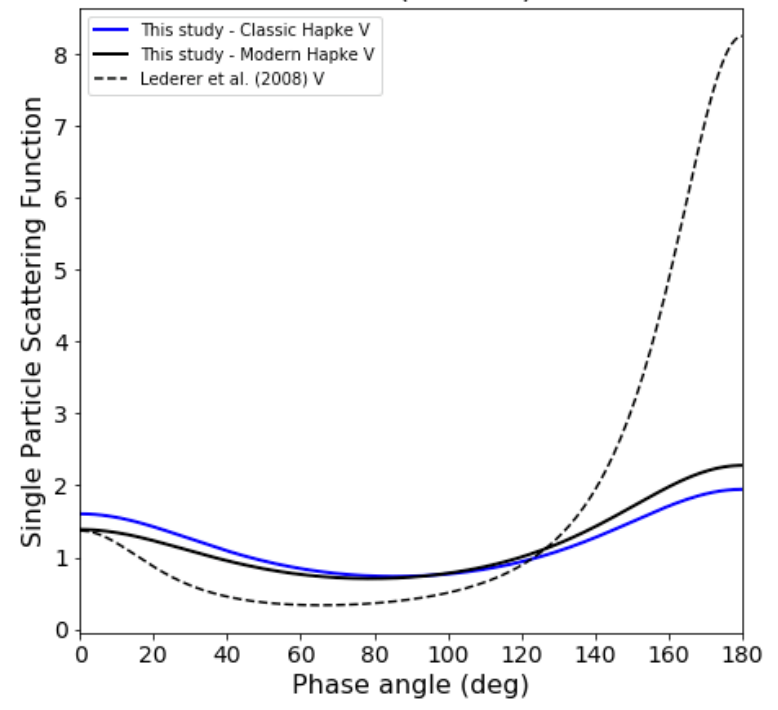

I filter (850 nm)

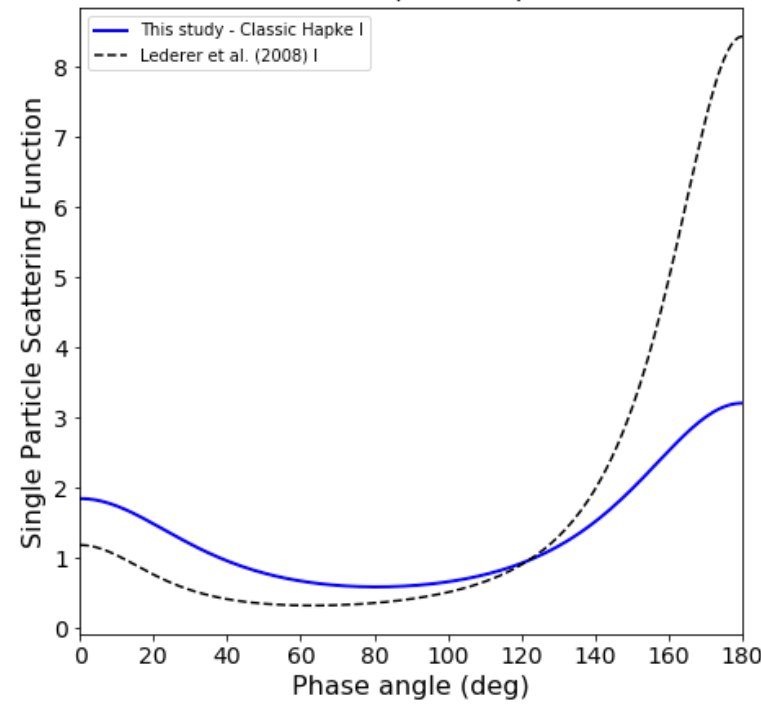

B filter $(440 \mathrm{~nm})$
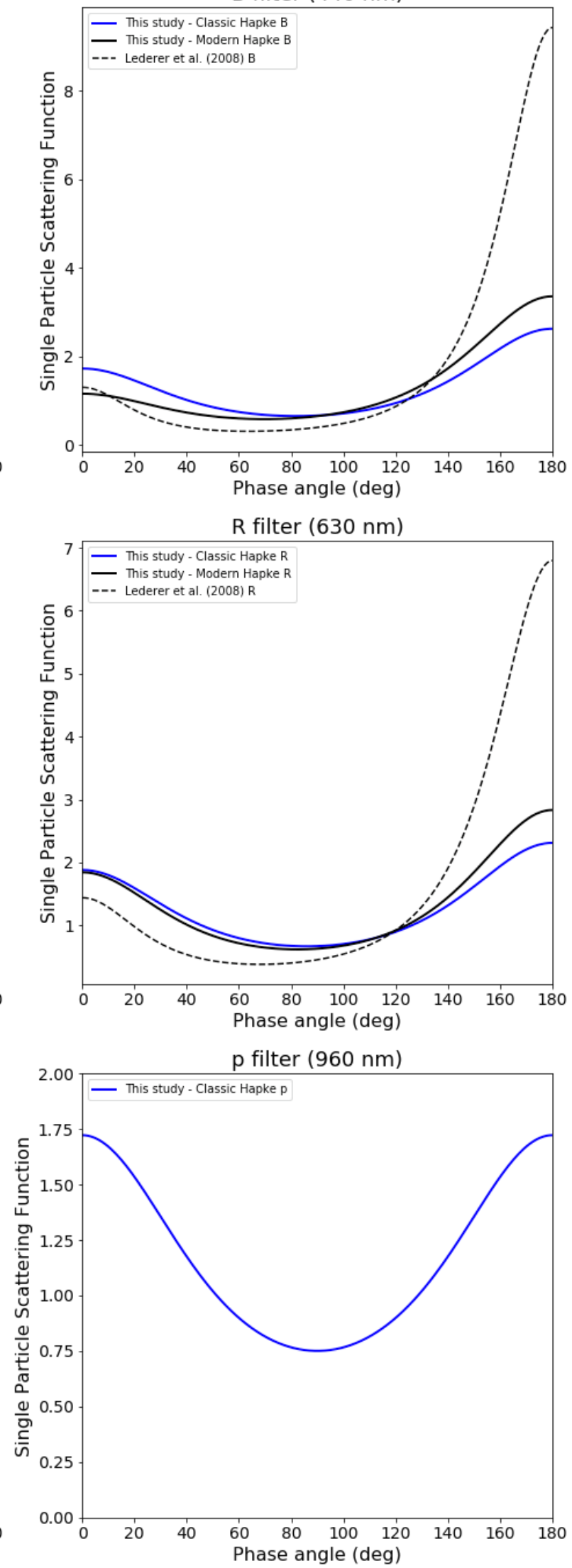
Figure 13. These graphs display the single-particle phase function for the Classic (solid black lines) and Modern (blue solid lines) Hapke modeling solutions compared to the scattering functions derived by Lederer et al. (2008) (dashed lines). Note that the single-particle phase functions from the Classic and Modern Hapke modeling results from this study are similar in depicting both a forward and a backward scattering component.
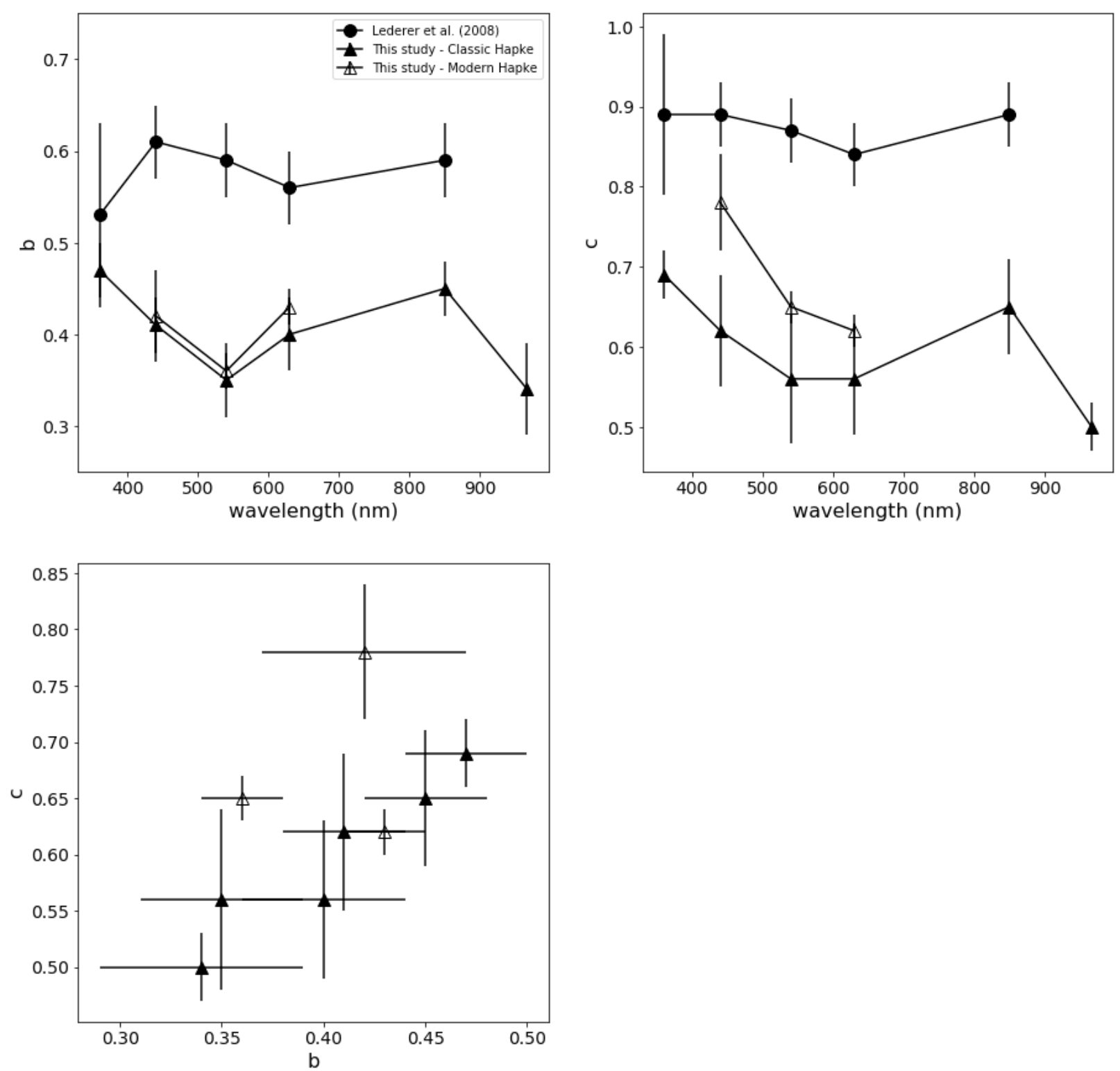

Figure 14. The top figures display the variability in the $b$ and $c$ parameters of single-particle phase function with wavelength for the Lederer et al. (2008) (black circles), the Classic Hapke (black triangles), and the Modern Hapke (open triangles) modeling results for the amplitude parameter (left) and the partition parameter (right) between forward and backward scattering. It is important to note that the results from this study display only modest variations with wavelength within the error bars. Comparisons of the two models used in this study (bottom) shows that the parameters are similar. 

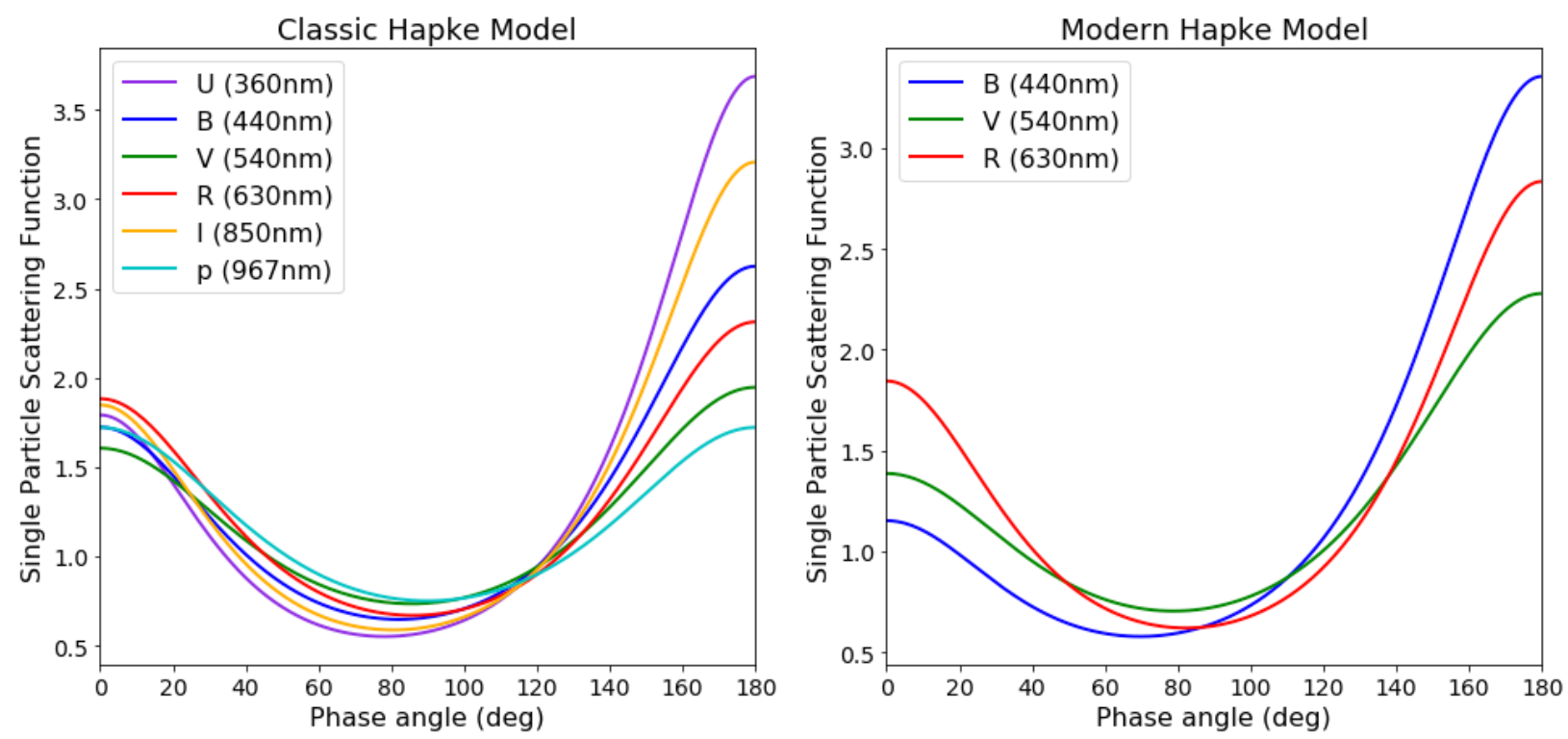

Figure 15. These figures compare the single-particle phase functions from the Classic Hapke Model (left) and the Modern Hapke model (right) for the wavelengths examined. The amplitude of the forward scattering direction varies with wavelength, but only provides a slightly stronger probability of scattering in the forward compared to backward direction.

\subsection{Comparisons with other asteroids/objects}

A number of asteroids that have been visited by spacecraft have been examined using Hapke's models, predominately the Classic Hapke Model. The derived geometric and Bond albedo, along with phase integral values, are provided in Table 8.

One comparison that can be made is an examination of the grain size distribution and porosity of their regolith based on the opposition parameters of the modeling results, or with the porosity term $\mathrm{K}$ if the data has been examined with the Modern Hapke model. Table 9 lists the $h_{s}$ values and corresponding porosity estimates for a number of these objects. The objects listed were examined with a form of the Classic Model, unless otherwise noted. The porosities listed are based on $\boldsymbol{r}_{l} / \boldsymbol{r}_{\boldsymbol{s}}$ ratios ranging from $200-$ 30,000 . The tables demonstrate a large range of porosities for a single object, and amongst the asteroids represented. The porosity values listed for Itokawa from this study are lower than those listed for average S-type asteroids. They are equivalent to those listed for S-type Lutetia, but lower than those for the remaining S-types in the table. In comparison to the C-type asteroids listed in the table, Itokawa has a higher porosity but overlaps with the estimated average for C-types.

Table 8. Equivalent V-filter Albedo and Phase Integral Values for Asteroids Visited by Spacecraft

\begin{tabular}{|c|c|c|c|c|c|}
\hline Object & Class & $\begin{array}{c}\text { Geometric } \\
\text { Albedo }\end{array}$ & $\begin{array}{c}\text { Bond } \\
\text { Albedo }\end{array}$ & $\begin{array}{c}\text { Phase } \\
\text { Integral }\end{array}$ & Source \\
\hline Itokawa & $\mathrm{S}$ & $0.27 \pm 0.02$ & $0.033 \pm 0.02$ & $0.13 \pm 0.02$ & This study (Classic) \\
\hline
\end{tabular}




\begin{tabular}{|c|c|c|c|c|c|}
\hline Itokawa & $\mathrm{S}$ & $0.19 \pm 0.02$ & $0.0209 \pm 0.01$ & $0.11 \pm 0.01$ & Lederer et al. (2008) \\
\hline Eros & $\mathrm{S}$ & $0.290 \pm 0.02$ & $0.12 \pm 0.02$ & $0.39 \pm 0.02$ & Domingue et al. (2002) \\
\hline Gaspra & $\mathrm{S}$ & $0.23 \pm 0.06$ & $0.12 \pm 0.03$ & $0.47 \pm 0.04$ & Helfenstein et al. (1994) \\
\hline Ida & $\mathrm{S}$ & $0.206 \pm 0.032$ & $0.081 \pm 0.017$ & $0.34 \pm 0.02$ & Helfenstein et al. (1996) \\
\hline Dactyl & $\mathrm{S}$ & $0.198 \pm 0.05$ & $0.073 \pm 0.021$ & $0.32 \pm 0.03$ & Helfenstein et al. (1996) \\
\hline Vesta $^{\S}$ & $\mathrm{V}$ & $0.38 \pm 0.04$ & $0.20 \pm 0.02$ & $0.53 \pm 0.10$ & Li et al. (2013) \\
\hline Bennu* $^{*}$ & $\mathrm{C}$ & $0.047 \pm 0.14$ & $0.015 \pm 0.007$ & $0.32 \pm 0.09$ & Takir et al. (2015) \\
\hline Ryugu & $\mathrm{C}$ & $0.047 \pm 0.003$ & $0.014 \pm 0.002$ & $0.32 \pm 0.03$ & Ishiguro et al. (2014) \\
\hline Mathilde & $\mathrm{C}$ & $0.047 \pm 0.005$ & $0.013 \pm 0.003$ & $0.28 \pm 0.05$ & Clark et al. (1999) \\
\hline Lutetia $^{\S}$ & $\mathrm{M}, \mathrm{C} ?$ & $0.194 \pm 0.002$ & $0.076 \pm 0.002$ & 0.255 & $\begin{array}{c}\text { Masoumzadeh et al. } \\
\end{array}$ \\
\hline Steins $^{\not b}$ & $\mathrm{E}$ & $0.39 \pm 0.02$ & $0.24 \pm 0.01$ & $0.59 \pm 0.04$ & Spjuth et al. (2012) \\
\hline
\end{tabular}

* Values are median of those listed from 3 different models, errors are largest errors listed from all values. \$Listed values are for $631.6 \mathrm{~nm}$, phase integral calculated from albedo relationships given in text. ${ }^{\mathscr{H}}$ Listed values are for $630 \mathrm{~nm}$.

Table 9. Comparison of Derived Regolith Properties

\begin{tabular}{|l|c|c|c|c|l|}
\hline \multicolumn{1}{|c|}{ Object } & Type & $\begin{array}{c}\lambda \\
(\mathbf{n m})\end{array}$ & $\mathbf{h}_{\mathbf{s}}$ & $\begin{array}{c}\boldsymbol{\rho}(\%) \\
\boldsymbol{\beta}=4\end{array}$ & \multicolumn{1}{c|}{ Source } \\
\hline Itokawa & $\mathrm{S}$ & 540 & 0.04 & $53.0-72.2$ & This study \\
\hline Itokawa & $\mathrm{S}$ & 540 & 0.11 & $17.4-40.8$ & This study \\
\hline Itokawa & $\mathrm{S}$ & 540 & 0.141 & $10.7-31.7$ & Lederer et al. (2008) \\
\hline Itokawa & $\mathrm{S}$ & 1570 & 0.01 & $85.3-92.2$ & Kitazato et al. (2008) \\
\hline Eros & $\mathrm{S}$ & 550 & 0.022 & $70.5-83.6$ & Domingue et al. (2002) \\
\hline Eros & $\mathrm{S}$ & 550 & 0.010 & $85.3-92.2$ & Li et al. (2004) \\
\hline Mathilde & $\mathrm{C}$ & 700 & 0.074 & $30.9-54.7$ & Clark et al. (1999) \\
\hline Gaspra & $\mathrm{S}$ & 560 & 0.06 & $38.6-61.3$ & $\begin{array}{l}\text { Helfenstein et al. } \\
\text { (1994) }\end{array}$ \\
\hline Ida & $\mathrm{S}$ & 560 & 0.020 & $72.8-85.0$ & $\begin{array}{l}\text { Helfenstein et al. } \\
\text { (1996) }\end{array}$ \\
\hline Bennu & $\mathrm{C}$ & 550 & 0.11 & $17.5-40.8$ & Takir et al. (2015) \\
\hline Lutetia & $\mathrm{S}$ & 649.2 & 0.041 & $52.3-71.6$ & $\begin{array}{l}\text { Masoumzadeh et al. } \\
\text { (2015) }\end{array}$ \\
\hline Steins & $\mathrm{E}$ & 630 & 0.027 & $65.2-80.2$ & Spjuth et al. (2012) \\
\hline Steins & $\mathrm{E}$ & 630 & 0.062 & $37.4-60.3$ & Spjuth et al. (2012) \\
\hline AnneFrank & $\mathrm{S}$ & 550 & 0.015 & $78.8-88.5$ & Hillier et al. (2011) \\
\hline Vesta & $\mathrm{V}$ & 554 & 0.07 & $32.9-56.5$ & Li et al. (2013) \\
\hline Ryugu & $\mathrm{C}$ & 550 & 0.050 & $45.2-66.5$ & Ishiguro et al. (2014b) \\
\hline Ave. S-type & $\mathrm{S}$ & 540 & 0.02 & $72.8-85.0$ & $\begin{array}{l}\text { Helefenstein and } \\
\text { Veverka (1989) }\end{array}$ \\
\hline Ave. C-type & $\mathrm{C}$ & 540 & 0.025 & $67.3-81.6$ & $\begin{array}{l}\text { Helefenstein and } \\
\text { Veverka (1989) }\end{array}$ \\
\hline
\end{tabular}

*Modern Hapke Model 
Hapke (2012b) described a relationship between the single-particle phase function parameters that could be correlated with regolith grain physical properties. This relationship, often referred to as the hockey stick relation, is shown in Figure 16 and includes the results from this study and from similar studies of other asteroids. The Itokawa results plot in a region characterized by particles with rough surfaces and/or low density of internal scatterers. This is in contrast with the Moon's parameters which are located in a region of moderate density of internal scatterers. The asteroids that are shown in this diagram were modeled under the assumption of a single-term scattering function, equivalent to setting the $c$ parameter to zero, thus they plot off the 'hockey stick' curve.

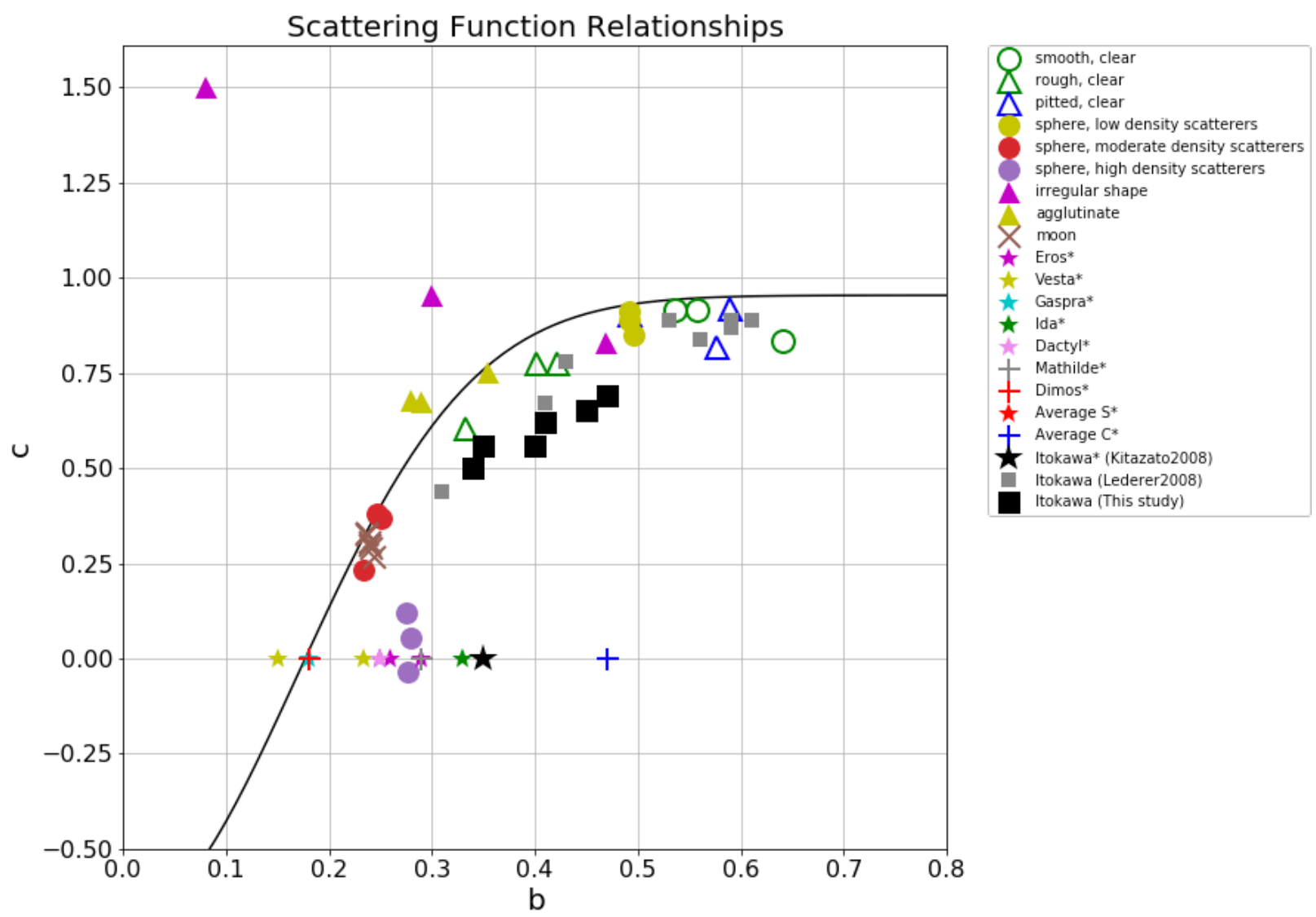

Figure 16. This graph shows the hockey stick relationship (Hapke, 2012b) correlating the Henyey-Greenstein single-particle phase function parameters with particle structures as measured in the laboratory by McGuire and Hapke (1995). Asteroids marked with an asterisk were modeled with a single-term Henyey-Greenstein phase function $(c=0)$.

\subsection{Comparison with the NIRS data}

The global photometric properties of Itokawa's surface in the near-infrared wavelength region $(0.85-2.1 \mu \mathrm{m})$ was derived by Kitazato et al. (2008) using data from Hayabusa's near-infrared spectrometer (NIRS). The NIRS data covers phase angles between $0.2^{\circ}-38.4^{\circ}$, which includes the opposition effect. Kitazato et al. (2008) modeled the NIRS data set using the Classic Hapke Model. Because the wavelength range of the NIRS 
data set overlaps in part with that of the AMICA data set, we can compare our parameters from the Classic Hapke Model with their results (Table 10).

Two different approaches were taken in modeling the NIRS and AMICA data sets. The data they modeled, while global in extent, involved disk-resolved measurements. In the case of the AMICA data, which included solely disk-integrated measurements, the value of $B_{0}$ was constrained to its theoretical limits due to the mathematical coupling in parameters that can be decoupled in disk-resolved measurements but not in disk-integrated. In addition, Kitazato et al. (2008) chose to use a single-term Henyey-Greenstein function to represent the single-particle phase function, whereas this study incorporated a two-term function. The values between the two forms of the single-particle phase function are related, such that $\mathrm{c}=0$ in the single-term function and the single-term parameter $\mathrm{g}$ is equivalent to $-\mathrm{b}$ (the value listed in Table 10).

Although they used the disk-resolved model with the different single-particle phase function, comparing the Hapke parameters of the AMICA and NIRS, opposition surge and scattering characteristics are very similar in terms of $B_{0}, h$, and $b$. However, we found that the SSA derived from the NIRS data modeling is much smaller than that from the AMICA data analyzed in this study. The alignment of NIRS and AMICA was well examined by Ishiguro et al. (2010), we can compare the spectra of NIRS and AMICA directly with simultaneously observed data. When we compare the spectra of the NIRS and the AMICA at the same place, (LAT, LON $\sim\left(-2.1^{\circ}, 256.1^{\circ}\right)$, at the same time on 2005-9-19, the AMICA spectra is $\sim 1.4$ times brighter than the NIRS spectra (Figure 17). One possible source of the difference in the absolute values in the spectra is in the different reflectance standards used to radiometrically calibrate each instrument. The NIRS calibration is based on a lunar reflectance standard and the AMICA calibration is based on standard star observations. This explains the much larger SSA for the AMICA photometry. It is noted that the larger $\theta$ value of the AMICA data may account for the larger SSA, although the roughness parameter cannot compensate this large SSA completely. However, when we multiplied the w values of NIRS with the $w$ ratio of the AMICA at p-band and the NIRS at $0.975 \mu \mathrm{m}$, we found that the relative shape of w spectrum is consistent with the ground observations (Figure 18).

Table 10. NIRS and AMICA Photometric Parameters at $976 \mu \mathrm{m}$ and p-filter.

\begin{tabular}{|c|c|c|}
\hline Model Parameter & NIRS (976 $\boldsymbol{\mu ~ m )}$ & AMICA (960 $\boldsymbol{\mu ~ m ) ~}$ \\
\hline $\mathrm{w}$ & $0.295 \pm 0.017$ & $0.59 \pm 0.03$ \\
\hline Bo & $0.870 \pm 0.016$ & $1.0 \pm 0.02$ \\
\hline $\mathrm{h}$ & $0.013 \pm 0.001$ & $0.02 \pm 0.02$ \\
\hline $\mathrm{b}$ & $0.350 \pm 0.013$ & $0.34 \pm 0.05$ \\
\hline $\mathrm{c}$ & 0.0 & $0.5 \pm 0.03$ \\
\hline$\theta$ & $26^{\circ} \pm 1^{\circ}$ & $38.3^{\circ}$ \\
\hline
\end{tabular}




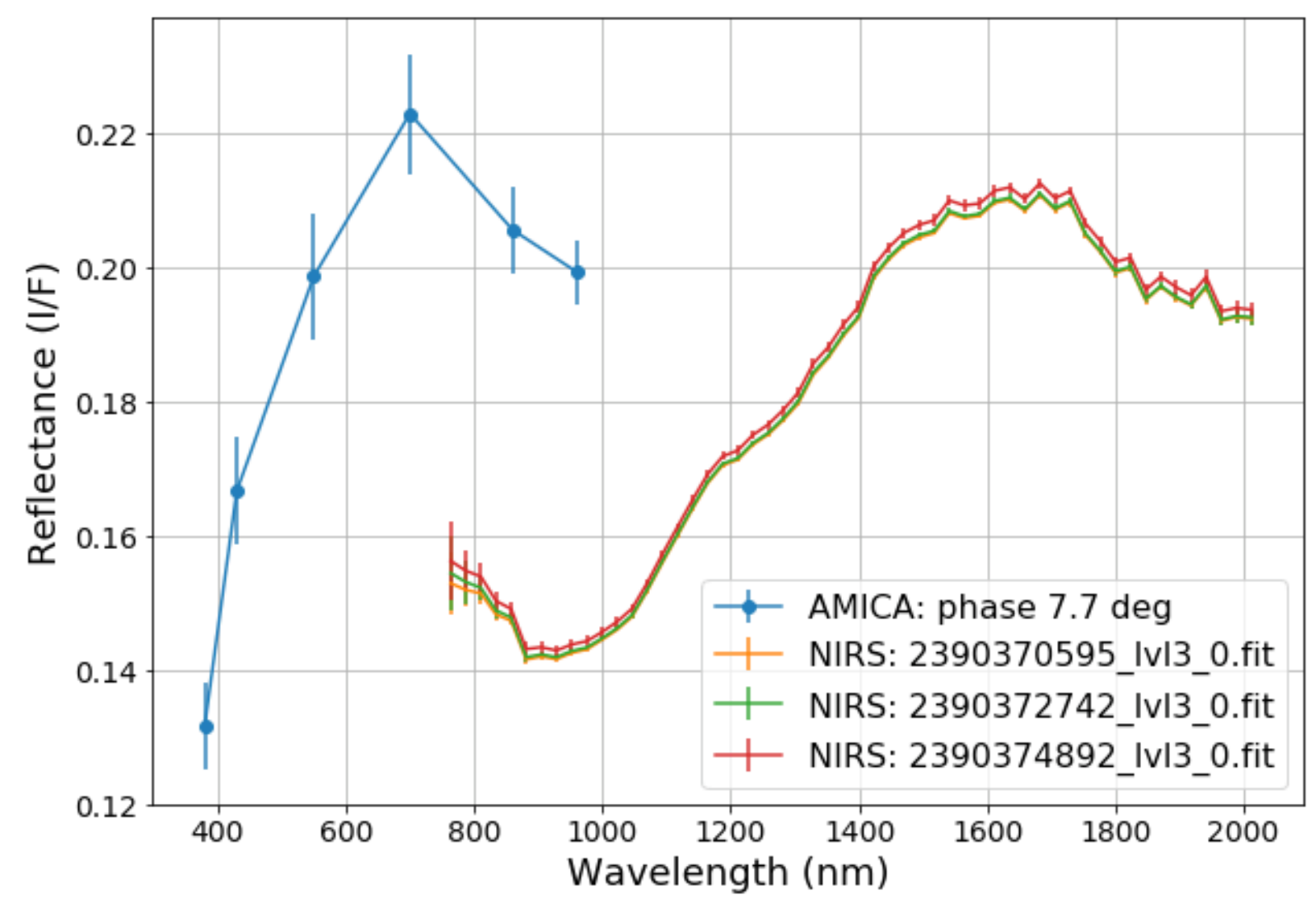

Figure 17. This shows the reflectance spectra of the NIRS and AMICA at the same observation point. The NIRS data were obtained simultaneously with AMICA observation and the error bars are acquired by the standard deviation of the data. The AMICA spectra are obtained from the dataset: st_2390397770_ul, st_2390417026_b, st_2390436248_v, st_2390455454_w, st_2390474726_x, st_2390513155_p. 


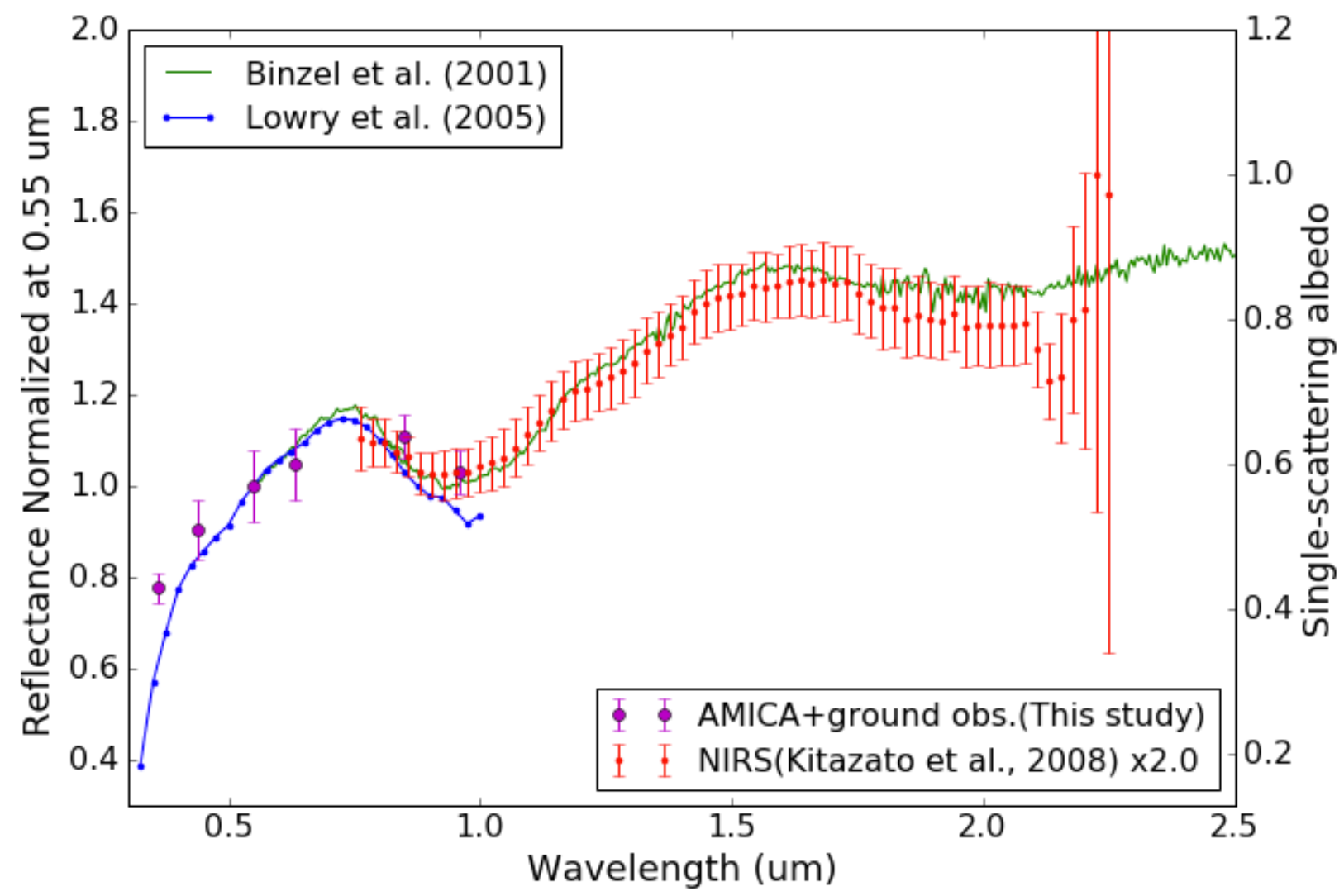

Figure 18. This shows the consistency between SSA of the AMICA and NIRS with spectra from ground observations (Binzel et al., 2001; Lowry et al., 2005). The NIRS data are multiplied by 2.0 to be the same value at the AMICA p-band. All the spectra are normalized at $0.55 \mu \mathrm{m}$.

\subsection{Spectral slope dependence on phase angle}

Here, for practical reasons, we assess the spectral slope dependence with phase angle based on the Classic Hapke Model results. Spectral reddening is defined as the increasing spectral slope with increasing phase angle value. This effect should be carefully taken into account when comparing the spectra acquired under different phase angle conditions. Phase reddening can mislead the interpretation of absorption bands and space weathering induced spectral slope changes. We calculated the model spectra for 5 different phase angles: $5^{\circ}, 10^{\circ}, 20^{\circ}, 30^{\circ}$, and $40^{\circ}$ (Figure 19). S-type asteroid compositions are mainly silicate rich material, containing such minerals as olivine and pyroxene. The depression at the p-band wavelength is indicative of an absorption band due to either olivine, pyroxene, or both. However, Figure 13 shows that the p-band absorption feature diminishes with increasing phase angle due to the phase reddening. Furthermore, the degree of space weathering or taxonomy classification is often defined by the slope of the visible spectrum (Bus and Binzel, 2001). Figure 20 shows the slope for each phase angle derived by least-square fitting from $0.36-0.96 \mu \mathrm{m}$. This suggests that for the case of Itokawa, the slope might be different by factor of 2 for phase angles $5^{\circ}$ and $40^{\circ}$. Thus, the phase reddening can easily cause confusion for comparison between different asteroids. 

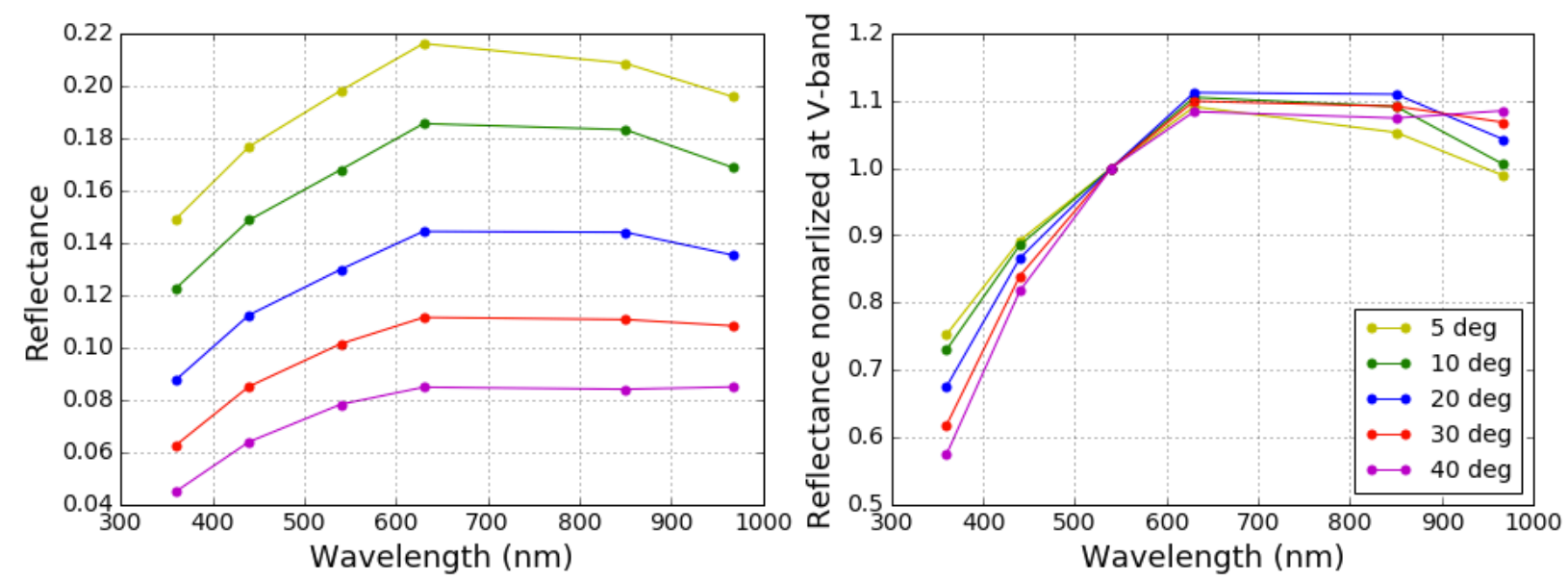

Figure 19. The variation of spectra with changing phase angle $5^{\circ}, 10^{\circ}, 20^{\circ}, 30^{\circ}$, and $40^{\circ}$. Reflectance (left) and reflectance normalized to $V$-band (right) are shown. The shorter wavelengths clearly show the reddening trend.

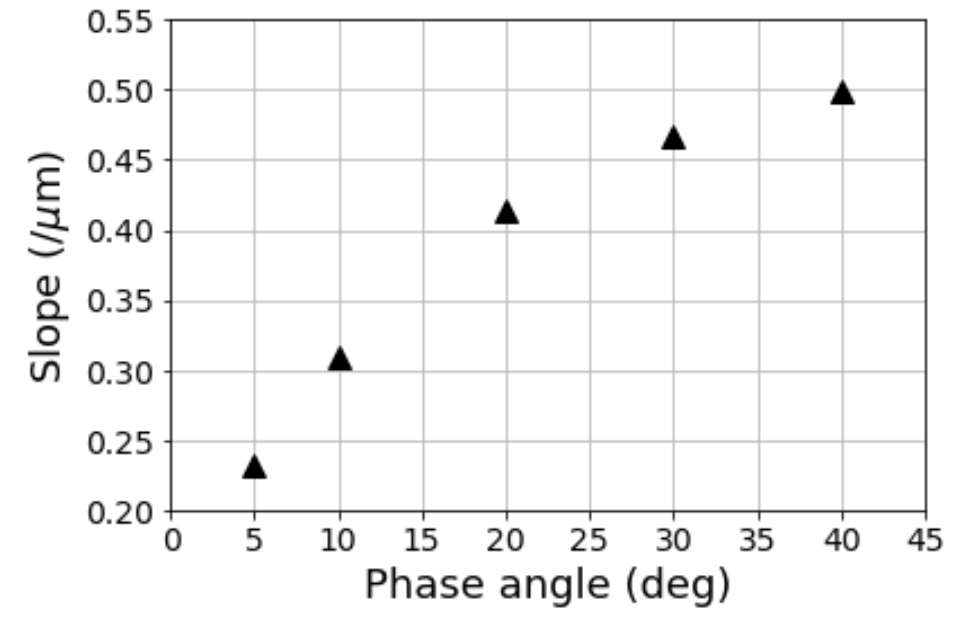

Figure 20. The slope derived by the least-square fitting from the spectra in Figure 19 is shown. The slope at phase angle $40^{\circ}$ is larger by a factor of $\sim 2$ than that at phase angle $5^{\circ}$.

\subsection{Conclusions}

The goals of this study were two-fold: 1) to test the viability of the photometric analysis of disk-integrated data to provide preliminary regolith information for planning landing or sampling operations during the early phase of a mission (where only low resolution and ground-based measurements are available), and 2) to understand the regolith characteristics that can be derived from such analyses. This study demonstrates the importance of both the unique opposition measurements provided by the AMICA instrument, and the need for large phase angle observations as provided by the ground-based observing campaigns. This study also demonstrates the accuracy and limitations in the ability to predict regolith characteristics from early phase, disk-integrated observations.

The AMICA instrument observed intensively around the opposition phase during the mission. This opposition data set, coupled with the ground-based observations, is sufficient to constrain the parameters that describe the opposition effect in both the Classic Hapke 
model and the Modern Hapke Model. The results regarding regolith grain size, grain size distribution, and porosity are commensurate with what is seen in the later mission phase, high-resolution images and returned-sample data. This approach can be used in the early mission phase, when high spatial resolution images are not available, to help prepare landing and sample return planning.

The unique point of the Modern Hapke Model is the surface compaction or porosity parameter $(k)$ that directly provides a surface porosity estimate. We obtained values of $k$ between $1-1.1$, which corresponds to porosities of $77-79 \%$. This high porosity is consistent with the radar observations by Ostro et al. (2004). The porosity derived from the Classic Hapke Model results are consistent with those from the Modern Hapke Model. However, the Classic Hapke Model can only provide an estimated porosity if an a prior grain size distribution is assumed. Using the porosity derived from the Modern Hapke model compaction parameter $k$, the grain size distribution can be derived from the shadow-hiding opposition width parameter. This provides the ratio of the largest to smallest grain within the regolith, but not the actual size. Using the empirical relationship between porosity and gravitational versus cohesive forces, a bond number can be measured which constrains the size range of the regolith. The results from this study suggest regolith grain sizes between $\mathrm{mm}$ to $\mathrm{cm}$, which is commensurate with the grain sizes observed in the Muses Sea region, but not the $\mathrm{cm}$ to $\mathrm{m}$ scale observed globally. The discrepancy is explained by the depth to which the photometric modeling is sensitive, $\mu \mathrm{m}$ to $\mathrm{mm}$. Thus, this technique underestimates the global grain sizes, but characterizes the smaller grain component of the surface.

Comparisons of the transport mean free path estimated from the Modern Hapke model opposition measurements and the returned sample analyses indicate that the surface reflectance at $\mu \mathrm{m}$ wavelengths is not sensitive to $\mathrm{nm}$-scale structures. The modeling results show that a $\sim 0.5 \mu \mathrm{m}$ photon will travel about twice its wavelength before encountering a scattering center. The single-particle phase function, however, predicts that once scattered the photon has a higher probability of being forward scattered. The hockey stick relationship between the single-particle phase function parameters (Hapke, 2012b), suggests Itokawa's surface grains are characterized by rough surfaces with a low density of inner scatterers. These results suggest that the scattering centers encountered at about every $\sim 1 \mu \mathrm{m}$ will direct the light in a 'more forward' direction. This is commensurate with scattering centers that correspond to mineral phase changes, changes in crystalinity, or pores. All of which are seen in the returned samples.

The V-band SSA, $w=0.57 \pm 0.05$, is slightly larger than that derived for Eros. This difference is caused by both compositional and particle size differences. There might be two explanations for this brightness of Itokawa; 1) fine particles cover the surface of gravels, 2) the surface of Itokawa is less space-weathered than Eros, 3) or both. The geometric albedo derived in this study for Itokawa is $0.27 \pm 0.02$, from both models. This is, within the error bars, equivalent to the geometric albedo derived for Eros $(0.29 \pm 0.02$, Domingue et al. 2002).

Comparisons of the photometric parameters obtained by NIRS (Kitazato et al., 2008) with those of this study (Table 15) show nearly identical surface characteristics for the opposition region (porosities and grain sizes) and the scattering functions (structural properties of the grains). However, the SSA derived from the AMICA and ground-based data 
set is approximately twice larger than that derived from the NIRS data set. In addition, the surface roughness derived from the AMICA dataset is larger (rougher) than that derived from the NIRS data set. The difference between the AMICA and NIRS surface roughness values are indicative of the differences in the regions of the surface each instrument samples. The NIRS data are descriptive of the equatorial region, where as the AMICA data set is influenced by Itokawa's irregular shape in addition to regional surface slopes. The roughness parameter for an irregular shaped asteroid must be interpret carefully, so that we need a local photometry to examine this large roughness value reflects a surface roughness or a global shape of an object.

We observe clear phase reddening on Itokawa. The slope differences with different phase angles can easily affect the taxonomy classification and the assessment of spectral characteristics, such as space weathering on Itokawa's surface. This emphasizes the importance of photometric standardization of color and spectral observations in interpreting Itokawa's surface properties.

\section{Acknowledgements}

The authors gratefully acknowledge Dr. Hapke and the anonymous reviewer for careful review. This work was supported by the JAXA/ISAS Hayabusa2 program, the Japan Society for Promotion of Science (JSPS) Core-to-Core Program's "International Network of Planetary Sciences", the JSPS KAKENHI (grant number 16H06719), NASA's Hayabusa2 participating scientist program (grant number NNX16AL34G), and NASA's Solar System Exploration Research Virtual Institute 2016 (SSERVI16) Cooperative Agreement (NNH16ZDA001N) for TREX (Toolbox for Research and Exploration).

\section{Appendix. A}

The Classic Hapke model for disk-integrated reflectance, $(I / F)_{c}$, is given by:

$$
\begin{gathered}
(I / F)_{c}=\left[\left\{\frac{w}{8}[(1+B(\alpha)) P(\alpha)-1]+\frac{r_{0}}{2}\left(1-r_{0}\right)\right\} \cdot\left\{1-\sin \left(\frac{\alpha}{2}\right) \tan \left(\frac{\alpha}{2}\right) \ln \left[\cot \left(\frac{\alpha}{4}\right)\right]\right\}\right. \\
\left.+\frac{2}{3} r_{0}\left(\frac{\sin (\alpha)+(\pi-\alpha) \cos (\alpha)}{\pi}\right)\right] S(\alpha, \theta) E q(3.1)
\end{gathered}
$$

where $w$ is the single-scattering albedo (SSA), $r_{0}$ is defined as

$$
r_{0}=\frac{1-\sqrt{1-w}}{1+\sqrt{1-w}}
$$

$\alpha$ is the phase angle, and $\theta$ is the surface roughness parameter. The $B(\alpha)$ is the opposition effect term defined as

$$
B(\alpha)=\frac{B_{S 0}}{1+\frac{\tan (\alpha / 2)}{h_{s}}}
$$


where $B_{S o}$ describes the amplitude of the opposition surge and $h_{s}$ describes the width of the opposition peak. In this model, the shadow-hiding mechanism (SHOE) is the sole source for the opposition effect (Hapke 1986, 2002, 2012a). The single-particle phase function, $P(\alpha)$, is provided by a two-term Henyey-Greenstein function of the form

$$
P(\alpha)=\frac{(1-c)\left(1-b^{2}\right)}{\left[1-2 b \cos (\alpha)+b^{2}\right]^{3 / 2}}+\frac{c\left(1-b^{2}\right)}{\left[1+2 b \cos (\alpha)+b^{2}\right]^{3 / 2}},
$$

where $b$ describes the amplitude of the forward and backward scattering peaks and $c$ is the partition parameter between forward and backward scattering. The surface roughness is incorporated in the $S(\alpha, \theta)$ term, where $\theta$ is the surface roughness parameter and is defined as the average slope over the resolution element of the detector (Hapke 1984). The derivation and mathematical expression for the surface roughness term can be found in Hapke $(1984,2012 \mathrm{a})$.

The Modern Hapke model for disk-integrated reflectance, $(I / F)_{\mathrm{m}}$, is given by:

$$
\begin{aligned}
(I / F)_{m}=\left(\frac{r_{0} A_{p}}{2 A_{p o}}\right)\left\{\left[\frac{(1+\gamma)^{2}}{4}\left\{\left[1+B_{S H O E}(\alpha)\right] P(\alpha)-1\right\}+\left[1-r_{0}\right]\right]\right. \\
\left.\cdot\left[1-\sin \frac{\alpha}{2} \tan \frac{\alpha}{2} \ln \left(\cot \frac{\alpha}{4}\right)\right]+\left(\frac{4}{3} r_{0}\right) \frac{\sin \alpha+(\pi-\alpha) \cos \alpha}{\pi}\right\}[1 \\
\left.+B_{C B O E}(\alpha)\right] K(\alpha, \theta), \quad E q(3.5)
\end{aligned}
$$

where $A_{p}$ is the physical albedo assuming a rough surface, $A_{p o}$ is the physical albedo assuming a smooth surface, and $\gamma=\sqrt{1-w}$ where $w$ is the single-scattering albedo. $B_{S H O E}$ is the shadow-hiding opposition term given by $B(\alpha)$ above, and $B_{C B O E}$ is the coherent-backscattering (CBOE) opposition term given by

$$
B_{\text {СВОЕ }}=\frac{B_{C 0}}{1+1.42 k}\left[\frac{1}{\left(1+\frac{1}{h_{c}} \tan \frac{\alpha}{2}\right)^{2}}\right]\left[1+\frac{1-\exp \left(-1.42 k \frac{1}{h_{c}} \tan \frac{\alpha}{2}\right)}{\frac{1}{h_{c}} \tan \frac{\alpha}{2}}\right], \quad E q(3.6)
$$

where $B_{C O}$ describes the amplitude of the coherent-backscatter opposition surge, $h_{c}$ describes the width of the coherent-backscatter opposition surge, and $k$ is the porosity of the surface. $P(\alpha)$ is the single-particle phase function shown above and $K(\alpha, \theta)$ is the surface roughness term. The derivation and mathematical expression for the Modern Hapke model and the surface roughness term can be found in Hapke $(2002,2008,2012 a)$.

\section{References}

Bhattacharya, S., Goswami, J., Lal, D., Patel, P., Rao, M., 1975. Lunar regolith and gas rich meteorites: Characterization based on particle tacks and grain size distribution. Proc. Lunar Sci. Conf. $6^{\text {th }}, 3509-3526$. 
Binzel, R. P., Rivkin, A. S., Bus, S. J., Sunshine, J. M., Burbine, T. H. (2001). MUSES - C target asteroid (25143) 1998 SF36: a reddened ordinary chondrite. Meteoritics \& Planetary Science, 36(8), 1167-1172.

Bonal, L., Brunetto, R., Beck, P., Dartois, E., Dionnet, Z., Djouadi, Z., Duprat, J., Füri, E., Kakazu, Y., Montagnac, G., Oudayer, P., Quirico, E., Engrand, C., 2015. Visible - IR and Raman microspectroscopic investigation of three Itokawa particles collected by Hayabusa: Mineralogy and degree of space weathering based on nondestructive analyses. Meteoritics \& Planetary Science, 50(9), pp. 1562-1576.

Bus, S. J., Binzel, R. P., 2002. Phase II of the small main-belt asteroid spectroscopic survey: A feature-based taxonomy. Icarus, 158, pp. 146-177.

Clark, B.E., Veverka, J., Helfenstein, P., Thomas, P.C., Bell III, J.F., Harch, A., 1999. NEAR Photometry of Asteroid 253 Mathidle. Icarus 140, 53 - 65.

Clark, B. E., Helfenstein, P., Bell, J.F., Peterson, C., Veverka, J., Izenberg, N. I., Domingue, D., Wellnitz, D., McFadden, L. (2002). NEAR Infrared Spectrometer Photometry of Asteroid 433 Eros, Icarus 155, Issue 1, 189-204, ISSN 0019-1035, http://dx.doi.org/10.1006/icar.2001.6748.

Domingue, D.L., Hapke, B.W., Lockwood, G.W., Thompson, D.T., 1991. Europa's phase curve - Implications for surface structure. Icarus 90, 30 - 42.

Domingue, D.L, Lockwood, G.W., Thompson, D.T. 1995. Surface textural properties of icy satellites: A comparison between Europa and Rhea. Icarus 115, Issue 2, 228 -249.

Domingue, D., M. Robinson, B. Carcich, J. Joseph, P. Thomas, and B. E. Clark 2002. Disk-integrated photometry of 433 Eros. Icarus 155, 205 - 219.

Domingue, D.L., Vilas, F., Holsclaw, G.M., Warell, J., Izenberg, N.R., Murchie, S.L., Denevi, B.W., Blewett, D.T., McClintock, W.E., Anderson, B.J., 2010. Whole-disk spectrophotometric properties of Mercury: Synthesis of MESSENGER and ground-based observations. Icarus 209, 101 - 124. doi:10.1016/j.icarus.2010.02.022.

Fujiwara, A., Kawaguchi, J., Yeomans, D. K., Abe, M., Mukai, T., Okada, T., Saito, J., Yano, H., Yoshikawa, M., Scheeres, D. J., Barnouin-Jha, O., Cheng, A. F., Demura, H., Gaskell, R. W., Hirata, N., Ikeda, H., Kominato, T., Miyamoto, H., Nakamura, A. M., Nakamura, R., Sasaki, S., Uesugi, K. (2006). The rubble-pile asteroid Itokawa as observed by Hayabusa. Science, 312(5778), 1330-1334.

Gaskell, R., Saito, J., Ishiguro, M., Kubota, T., Hashimoto, T., Hirata, N., Abe, S., Barnouin-Jha, O. S., and Scheeres, D., Gaskell Itokawa Shape Model V1.0. HAY-A-AMICA-5-ITOKAWASHAPE-V1.0. NASA Planetary Data System, 2008.

Gehrels, T. 1956. Photometric Studies of Asteroids. V. The Light-Curve and Phase Function of 20 Massalia. Astrophysical Journal 123, pp.331-338.

Gundlach, B., Blum, J. 2012. A new method to determine the grain size of planetary regolith. Icarus 223, $479-492$.

Hapke, B., 1981. Bidirectional reflectance spectroscopy. 1. Theory. J. Geophys. Res. 68, 4571 $-4586$.

Hapke, B., 1984. Bidirectional reflectance spectroscopy 3. Correction for macroscopic roughness. Icarus 59, $41-59$.

Hapke, B., 1986. Bidirectional reflectance spectroscopy 4. The extinction coefficient and the opposition effect. Icarus 67, $264-280$.

Hapke, B. W., Nelson, R. M., Smythe, W. D., 1993. The opposition effect of the moon: the 
contribution of coherent backscatter. Science, 260(5107), 509-511.

Hapke, B., 2002. Bidirectional reflectance spectroscopy. 5. The coherent backscatter opposition effect and anisotropic scattering. Icarus 157, $523-534$.

Hapke, B., 2008. Bidirectional reflectance spectroscopy. 6. Effects of porosity. Icarus 195, $918-926$.

Hapke, B., 2012a. Theory of Reflectance and Emittance Spectroscopy. Cambridge University Press, NY, $2^{\text {nd }}$ Edition, 513 pp.

Hapke, B. 2012b. Bidirectional reflectance spectroscopy 7 The single particle phase function hockey stick relation. Icarus 221, 1079 - 1083.

Harries, D., Yakame, S., Karouji, Y., Uesugi, M., Langenhorst, F., 2016. Secondary submicrometer impact cratering on the surface of asteroid 25143 Itokawa, Earth and Planetary Science Letters 450, pp. 337-345, ISSN 0012-821X, https://doi.org/10.1016/j.epsl.2016.06.033.

Helfenstein, P. (1988). The geological interpretation of photometric surface roughness. Icarus, 73(3), 462-481.

Helfenstein, P., Veverka, J., Thomas, P.C., Simonelli, D.P., Lee, P., Klaasen, K, Johnson, T.V., Breneman, H., Head, J.W., Murchie, S., 1994. Galileo photometry of Asteroid 951 Gaspra, Icarus, 107, $37-60$.

Helfenstein, P., Shepard, M. K., 2011. Testing the Hapke photometric model: Improved inversion and the porosity correction. Icarus, 215(1), 83-100.

Helfenstein, P., Veverka, J., Thomas, P.C., Simonelli, D.P., Klaasen, K., Johnson, T.V., Fanale, F., Granahan, J., McEwen, A.S., Belton, M., Chapman, C, 1996. Galileo photometry of Asteroid 243 Ida, Icarus, 120, 48 - 65.

Ishiguro, M., Nakamura, R., Tholen, D. J., Hirata, N., Demura, H., Nemoto, E., Nakamura, A. M., Higuchi, Y., Sogame, A., Yamamoto, A., Kitazato, K., Yokota, Y., Kubota, T., Hashimoto, T., Saito, J. (2010). The Hayabusa Spacecraft Asteroid Multi-band Imaging Camera (AMICA), Icarus 207, Issue 2, 714-731, ISSN 0019-1035, http://dx.doi.org/10.1016/j.icarus.2009.12.035.

Ishiguro, M., 2014a. Scattered light correction of Hayabusa/AMICA data and quantitative spectral comparisons of Itokawa. Publications of the Astronomical Society of Japan, 66(3).

Ishiguro, M., Kuroda, D., Hasegawa, S., Kim, M. J., Choi, Y. J., Moskovitz, N., Abe, S., Pan, K. S., Takahashi, J., Takagi, Y., Arai, A., Tokimasa, N., Hsieh, H. H., Thomas-Osip, J. E., Abe, M., Yoshikawa, M., Urakawa, S., Hanayama, H., Sekiguchi, T., Wada, K., Sumi, T., Tristram, P. J., Furusawa, K., Abe, F., Fukui, A., Nagayama, T., Warjurkar, D. S., Rau, A., Greiner, J., Schady, P., Knust, F., Usui, F., Müller, T. G., 2014b. Optical properties of (162173) 1999 JU3: in preparation for the JAXA Hayabusa 2 sample return mission. The Astrophysical Journal, 792(1), 74.

Kaasalainen, S. (2003). Laboratory photometry of planetary regolith analogs-I. Effects of grain and packing properties on opposition effect. Astronomy \& Astrophysics, 409(2), 765-769.

Kitazato, K., Clark, B. E., Abe, M., Abe, S., Takagi, Y., Hiroi, T., Barnouin-Jha, O. S., Abell, P. A., Lederer, M. S., Vilas, F., (2008). Near-infrared spectrophotometry of Asteroid 25143 Itokawa from NIRS on the Hayabusa spacecraft, Icarus 194, 1, 137-145, ISSN 0019-1035, http://dx.doi.org/10.1016/j.icarus.2007.08.029.

Kiuchi, M., Nakamura, A.M., 2014. Relationship between regolith particle size and porosity 
on small bodies. Icarus 239, $291-293$.

Koga, S. C., Sugita, S., Kamata, S., Ishiguro, M., Hiroi, T., Tatsumi, E., Sasaki, S. (2018). Spectral decomposition of asteroid Itokawa based on principal component analysis. Icarus 299, pp. 386-395.

Lederer, S.M., Domingue, D.L., Vilas, F., Abe, M., Farnham, T.L., Jarvis, K.S., Lowry, S.C., Ohba, Y., Weissman, P.R., French, L.M., Fukai, H., Hasegawa, S., Ishiguro, M., Larson, S.M., Takagi, Y. 2005. Physical characteristics of Hayabusa target Asteroid 25143 Itokawa. Icarus 173, Issue 1, 153 - 165.

Lederer, S.L., Domingue, D.L., Thomas-Osip, J.E., Vilas, F., Osip, D.J., Leeds, S.L., Jarvis K.S. 2008. The 2004 Las Campanas/Lowell Observatory campaign II. Surface properties of Hayabusa target Asteroid 25143 Itokawa inferred from Hapke modeling. Earth Planets Space 60, $49-59$.

Li, J., A'Hearn, M. F., McFadden, L. A., 2004. Photometric analysis of Eros from NEAR data. Icarus, 172(2), pp. 415-431.

Li, J. Y., Le Corre, L., Schröder, S. E., Reddy, V., Denevi, B. W., Buratti, B. J., Mottola, S., Hoffmann, M., Gutierrez-Marques, P., Nathues, A., Russell, C. T., Raymond, C. A., 2013. Global photometric properties of Asteroid (4) Vesta observed with Dawn Framing Camera. Icarus, 226(2), pp. 1252-1274.

Lowry, S. C., Weissman, P. R., Hicks, M. D., Whiteley, R. J., Larson, S., 2005. Physical properties of Asteroid (25143) Itokawa-Target of the Hayabusa sample return mission. Icarus, 176(2), pp. 408-417.

Lowry, S. C., Weissman, P. R., Duddy, S. R., Rozitis, B., Fitzsimmons, A., Green, S. F., Hicks, M. D., Snodgrass, C., Wloters, S. D., Chesley, S. R., Pittichová, J., van Oers, P., 2014. The internal structure of asteroid (25143) Itokawa as revealed by detection of YORP spin-up. Astronomy \& Astrophysics, 562, A48.

Magrin, S., La Forgia, F., Pajola, M., Lazzarin, M., Massironi, M., Ferri, Da Deppo, D., Barbieri, C., Sierks, H., The OSIRIS Team. (2012). (21) Lutetia spectrophotometry from Rosetta-OSIRIS images and comparison to ground-based observations. Planetary and Space Science, 66(1), 43-53, https://doi.org/10.1016/j.pss.2011.10.001.

Masoumzadeh, N., Boehnhardt, H., Li, J.-Y., Vincent, J.-B., 2015. Photometric analysis of Asteroid (21) Lutetia from Rosetta-OSIRIS images. Icarus 257, 239 - 250.

Mazrouei, S., Daly, M. G., Barnouin, O. S., Ernst, C. M., DeSouza, I., 2014. Block distributions on Itokawa. Icarus, 229, 181-189.

McGuire, A., Hapke, B., 1995. An experimental study of light scattering by large, irregular particles. Icarus $113,134-155$.

Michikami, T., Nakamura, A. M., Hirata, N., Gaskell, R. W., Nakamura, R., Honda, T., Honda, C., Hiraoka, K., Saito, J., Demura, H., Ishiguro, M., Miyamoto, H., 2008. Size-frequency statistics of boulders on global surface of asteroid 25143 Itokawa. Earth, planets and space, 60(1), pp. 13-20.

Miyamoto, H., Yano, H., Scheeres, D.J., Abe, S., Barnouin-Jha, O., Cheng, A.F., Demura, H., Gaskell, R.W., Hirata, N., Ishiguro, M., Michikami, T., Nakamura, A.M>, Nakamura, R., Saito, J., Sasaki, S., 2007. Regolith Migraation and Sorting on Asteroid Itokawa. Science 316, $1011-1014$.

Nagao, K., Okazaki, R., Nakamura, T., Miura, Y. N., Osawa, T., Bajo, K. I., Matsuda, S., Ebihara, M., Ireland, T. R., Kitajima, F., Naraoka, H., Noguchi, T., Tsuchiyama, A., Yurimoto, H., Zolensky, M. E., Uesugi, M., Shirai, K., Abe, M., Yada, T., Ishibashi, Y., Fujiwara, A., Mukai, 
T., Ueno, M., Okada, T., Yoshikawa, M., Kawaguchi, J., (2011). Irradiation history of Itokawa regolith material deduced from noble gases in the Hayabusa samples. Science, 333(6046), 1128-1131.

Noguchi, T., Kimura, M., Hashmoto, T., Konno, M., Nakamura, T., Zolensky, M.E., Okazaki, R., Tnanka, M., Tsuchiyama, A., Nakato, A., Ogami, T., Ishida, H., Sagae, R., Abe, M., yada, T., Mukai, T., Ueno, M., Okada, T., Shirai, K., Ishibashi, Y., 2013. Space weathered rims found on the surfaces of the Itokawa dust particles, Meteoritics \& Planetary Science 1 - 27, doi: $10.1111 /$ maps.12111

Nakamura, T., Noguchi, T., Tanaka, M., Zolensky, M. E., Kimura, M., Tsuchiyama, A., Nakato, A., Ogami, T., Ishida, H., Uesugi, M., Yada, T., Shirai, K., Fujimura, A., Okazaki, R., Sandford, S. A., Ishibashi, Y., Abe, M., Okada, T., Ueno, R., Mukai, T., Yoshikawa, M., Kawaguchi, J., 2011. Itokawa dust particles: a direct link between S-type asteroids and ordinary chondrites. Science, 333, pp. 1113-1116.

Nakamura, T., Nakato, A., Ishida, H., Wakita, S., Noguchi, T., Zolensky, M. E., Tanaka, M., Kimura, M., Tsuchiyama, A., Ogami, T., Hashimoto, T., Konno, M., Uesugi, M., Yada, T., Shirai, K., Fujiwara, A., Okazaki, R., Sanford, S. A., Ishibashi, Y., Abe, M., Okada, T., Ueno, M., Kawaguchi, J., 2014. Mineral chemistry of MUSES - C Regio inferred from analysis of dust particles collected from the first - and second - touchdown sites on asteroid Itokawa. Meteoritics \& Planetary Science, 49(2), pp. 215-227.

Ostro, S.J., Benner, L.A.M., Nolan, M.C., Magri, C., Giorgini, J.D., Scheeres, D.J., Broschart, S.B., Kaasalainen, M., Vokrouhlicky, D., Chesley, S.R., Margot, J.-L., Jurgens, R.F., Rose, R., Yeomans, D.K., Suzuku, S., de Jong, E.M., 2004. Radar observations of asteroid 25143 Itokawa (1998 SF36). Meteorit. Planets. Sci. 39, 407 - 424.

Saito, J., Miyamoto, H., Nakamura, R., Ishiguro, M., Michikami, T., Nakamura, A. M., Demura, H., Sasaki, S., Hirata, N., Honda, C., Yamamoto, A., Yokota, Y., Fuse, T., Tholen, D . J., Gaskell, R. W., Hashimoto, T., Kubota, T., Higuchi, Y., Nakamura, T., Smith, P., Hiraoka, K., Honda, T., Kobayashi, S., Furuya, M., Matsumoto, N., Nemoto, E., Yukishita, A., Kitazato, K., Dermawan, B., Sogame, A., Terazono, J., Shinohara, C., Akiyama, H., 2006. Detailed images of asteroid 25143 Itokawa from Hayabusa. Science, 312(5778), 1341-1344.

Saito, J., Nakamura, T., Akiyama, H., Demura, H., Dermawan, B., Furuya, M., Fuse, T., Gaskell, R., Hashimoto, T., Higuchi, Y., Hiraoka, K., Hirata, N., Honda, C., Honda, T., Ishiguro, M., Kitazato, K., Kobayashi, S., Kubota, T., Matsumoto, N., Michikami, T., Miyamoto, H., Nakamura, A., Nakamura, R., Nemoto, E., Sasaki, S., Shinohara, C., Smith, P., Sogame, A., Terazono, J., Tholen, D., Yamamoto, A., Yokota, Y., Yoshida, F., and Yukishita, A., Hayabusa AMICA V1.0. HAY-A-AMICA-3-HAYAMICA-V1.0. NASA Planetary Data System, 2010.

Sánchez, P., \& Scheeres, D. J. (2014). The strength of regolith and rubble pile asteroids. Meteoritics \& Planetary Science, 49(5), 788-811.

Scheeres, D. J., Hartzell, C. M., Sánchez, P., Swift, M., 2010. Scaling forces to asteroid surfaces: The role of cohesion. Icarus, 210(2), 968-984.

Schröder, S. E., Mottola, S., Keller, H. U., Raymond, C. A., Russell, C. T., 2013. Resolved photometry of Vesta reveals physical properties of crater regolith. Planetary and Space Science, 85, 198-213.

Seeliger, H. (1895). Theorie der Beleuchtung staubformiger kosmischen Masses insbesondere des Saturinges. Abh. Bayer. Acad. Wiss. Math.-Naturwiss. Kl, 18, pp. 1-72. 
Shepard, M. K., Campbell, B. A. (1998). Shadows on a planetary surface and implications for photometric roughness. Icarus, 134(2), 279-291.

Shepard, M. K., Helfenstein, P., 2007. A test of the Hapke photometric model. Journal of Geophysical Research: Planets, 112(E3).

Souchon, A.L., Pinet, P.C., Chevrel, S.D., Daydou, Y.H., Baratoux, D., Kurita, K., Shepard, M.K., Helfenstein, P. 2011. An experimental study of Hapke's modeling of natural granular surface samples. Icarus 215, 313 - 331.

Spjuth, S., Jorda, L., Lamy, P.L., Keller, H.U., Li, J.-Y., 2012. Disk-resolved photometry of Asteroid (2867) Steins. Icarus 221, 1101 - 1118.

Suzuki, H., Yamada, M., Kouyama, T., Tatsumi, E., Kameda, S., Honda, R., Sawada, H., Ogawa, N., Morota, T., Honda, C., Sakatani, N., Hayakawa, M., Yokota, Y., Yamamoto, Y., Sugita, S. (2018). Initial inflight calibration for Hayabusa2 optical navigation camera (ONC) for science observations of asteroid Ryugu. Icarus, 300, 341-359.

Takir, D., Clark, B.E., d'Aubigny, C.D., Hergenrother, C.W., Li, J.-Y., Lauretta, D.S., Binzel, R.P., 2015. Photometric models of disk-integrated observations of the OSIRIS-Rex target Asteroid (101955) Bennu. Icarus, 252, 393 - 399.

Tancredi, G., Roland, S., Bruzzone, S., 2015. Distribution of boulders and the gravity potential on asteroid Itokawa. Icarus 247, 279 - 290.

Tatsumi, E., Sugita, S. (2017). Cratering efficiency on coarse-grain targets: implications for the dynamical evolution of asteroid 25143 Itokawa. Icarus, 300, pp. 227-248.

Tsuchiyama, A., Uesugi, M., Matsushima, T., Michikami, T., Kadono, T., Nakamura, T., Uesugi, K., Nakano, T., Sanford, S. A., Noguchi, R., Matsumoto, T., Masuno, J., Nagano, T., Imai, Y., Takeuchi, A., Suzuki, Y., Ogami, T., Katagiri, J., Ebihara, M., Ireland, T. R., Kitajima, F., Nagao, K., Naraoka, H., Noguchi, T., Okazaki, R., Yurimoto, H., Zolensky, M. E., Mukai, T., Abe, M., Yada, T., Fujimura, A., Yoshikawa, M., Kawaguchi, J., 2011. Three-dimensional structure of Hayabusa samples: origin and evolution of Itokawa regolith. Science, 333(6046), pp. 1125-1128.

Yano, H., Kubota, T., Miyamoto, H., Okada, T., Scheeres, D., Takagi, Y., Yoshida, K., Abe, M., Abe, S., Barnouin-Jha, O., Fujiwara, A., Hasegawa, S., Hashimoto, T., Ishiguro, M., Kato, M., Kawaguchi, J., Mukai, T., Saito, J., Sasaki, S., Yoshikawa, M., 2006. Touchdown of the Hayabusa Spacecraft at the Muses Sea on Itokawa. Science 312, 1350 - 1353.

Yu, A. B., Feng, C. L., Zou, R. P., Yang, R. Y. (2003). On the relationship between porosity and interparticle forces. Powder Technology, 130(1), 70-76.

Zellner, B., Tholen, D. J., Tedesco, E. F. (1985). The eight-color asteroid survey: Results for 589 minor planets. Icarus, 61(3), 355-416. 